\title{
Answer Set Programming based on Propositional Satisfiability
}

\author{
Enrico Giunchiglia ${ }^{1}$, Yuliya Lierler ${ }^{2}$ and Marco Maratea ${ }^{1,3}$ \\ 1 STAR-Lab, DIST, University of Genova \\ viale Francesco Causa, 13 - 16145 Genova, Italy \\ \{enrico,marco\}@dist. unige. it \\ ${ }^{2}$ Institut für Informatik, Erlangen-Nürnberg-Universität \\ Haberstr. 2, Erlangen, Germany \\ yuliya@informatik. uni-erlangen. de \\ ${ }^{3}$ Department of Mathematics, University of Calabria \\ via P. Bucci, Cubo $30 b-87036$ Rende (CS), Italy
}

\begin{abstract}
Answer Set Programming (ASP) emerged in the late 1990s as a new logic programming paradigm which has been successfully applied in various application domains. Also motivated by the availability of efficient solvers for propositional satisfiability (SAT), various reductions from logic programs to SAT were introduced in the past. All these reductions either are limited to a subclass of logic programs, or introduce new variables, or may produce exponentially bigger propositional formulas.

In this paper, we present a SAT-based procedure, called ASP-SAT, that $(i)$ deals with any (non disjunctive) logic program, (ii) works on a propositional formula without additional variables (except for those possibly introduced by the clause form transformation), and (iii) is guaranteed to work in polynomial space. From a theoretical perspective, we prove soundness and completeness of ASP-SAT. From a practical perspective, we have $(i)$ implemented ASP-SAT in CMODELS, (ii) extended the basic procedures in order to incorporate the most popular SAT reasoning strategies, and (iii) conducted an extensive comparative analysis involving also other state-of-the-art answer set solvers. The experimental analysis shows that our solver is competitive with the other solvers we considered, and that the reasoning strategies that work best on "small but hard" problems are ineffective on "big but easy" problems and vice versa.
\end{abstract}

Keywords: Answer Set Programming, Propositional Satisfiability

\section{Introduction}

Answer Set Programming (ASP) emerged in the late 1990s as a new logic programming paradigm (Marek and Truszczynski, 1999; Niemelä, 1999), and has been successfully applied in various domains including space shuttle control (Nogueira et al., 2001), planning (Lifschitz et al., 1999), and the design and implementation of query answering systems (Baral and Scherl, 2004). Syntactically, ASP programs look like Prolog programs, but they are treated by rather different computational

(c) 2006 Kluwer Academic Publishers. Printed in the Netherlands. 
mechanisms. Indeed, ASP systems like CMODELS (Lierler and Lifschitz,

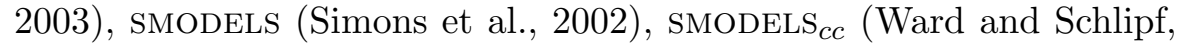
2004), DLV (Leone et al., 2005), and ASSAT (Lin and Zhao, 2002; Lin and Zhao, 2004) interpret logic programs via the answer set semantics (Gelfond and Lifschitz, 1988; Gelfond and Lifschitz, 1991). The goal is to find the "models" (called answer sets) of the program, and not to evaluate whether a query is true or not, as in standard Prolog systems. The ASP approach is thus similar to propositional satisfiability checking, where propositional formulas encode the problem and models of the formula correspond to the solutions of the problem.

Propositional satisfiability (SAT) is one of the most intensely studied fields in Artificial Intelligence and Computer Science. Various procedures that can deal with thousands of variables are now available (see, e.g., (Le Berre and Simon, 2003)). Also motivated by the availability of efficient SAT solvers (such as SATZ (Li and Anbulagan, 1997) and MCHAFF (Moskewicz et al., 2001)), various reductions from logic programs to SAT were introduced in the past. The most popular of such reductions is Clark's completion (Clark, 1978). Fages (1994) showed that if a logic program is "tight" then its answer sets are in one-toone correspondence with the models of its Clark's completion. From a theoretical point of view, Fages' result was then generalized to include programs with infinitely many rules (Lifschitz, 1996), programs tight "on their completion models" (Babovich et al., 2000), programs with nested expressions in the bodies of the rules (Erdem and Lifschitz, 2001), and disjunctive programs (Lee and Lifschitz, 2003). From a practical point of view, computation of answer sets for tight programs via Clark's completion and SAT solving has been first implemented in CMODELS, and has been also shown to be effective on many classes of problems. Still, these results do not apply to the whole class of logic programs. In general, it is well known that each answer set corresponds to a model of its completion, but the converse is in general not true (Marek and Subrahmanian, 1989).

Ben-Eliyahu and Dechter (1996) gave a translation from a class of disjunctive logic programs to SAT: Their translation may need $O\left(n^{2}\right)$ new variables and $O\left(n^{3}\right)$ new clauses, where $n$ is the number of atoms in the logic program. Lin and Zhao (2003a) introduced a translation which needs the introduction of $O\left(n^{2}+m\right)$ new variables and $O(n \times m)$ new clauses, where $m$ is the number of rules in the logic program. Janhunen (2004) presented an optimized encoding which is sub-quadratic in both size and number of atoms. Lin and Zhao (2004) report that the grounding of a program corresponding to the computation of a Hamiltonian path in a complete graph with 50 nodes, produces a program with 5000 atoms and 240000 rules, and in a complete graph of 60 nodes 
produces a program with 7000 atoms and 420000 rules. For problems like these, the number of variables or clauses in the resulting formula may become prohibitive.

The only reduction to SAT which does not need extra variables has been proposed by Lin and Zhao $(2002,2004)$. The drawback of this reduction is that it may blow up in space, i.e., the resulting number of clauses can be exponential. This is not by chance. A recent result by Lifschitz and Razborov (Lifschitz and Razborov, 2004) shows that assuming $P \nsubseteq N C^{1} /$ poly, a conjecture from computational complexity theory widely believed to be true - whenever we try to translate a logic program to a set of clauses

- either we have to introduce new variables,

- or an exponential blow up may occur.

Despite the potential exponential blow up, system ASSAT based on such a reduction outperforms state-of-the-art ASP systems like SMODELS and DLV on many interesting problems.

In this paper we present a procedure, called ASP-SAT, that

1. deals with any (not necessarily tight) logic program,

2. works on a propositional formula without additional variables (except for those possibly introduced by the clause form transformation), and

3. is guaranteed to work in polynomial space.

From a theoretical perspective, we prove the soundness and completeness of ASP-SAT. We also show how to extend this basic procedure in order to compute all answer sets still working in polynomial space.

From a practical perspective, we have implemented ASP-SAT in CModels. We call the resulting system CModels2. Given the SATbased nature of our procedure, we have been able to implement — with a relatively small effort - several search strategies and heuristics which have been shown effective in the SAT literature. Then, we experimentally analyze which combinations of reasoning strategies work best on which problems. In particular,

- We implemented various "look-ahead" strategies (used while descending the search tree); "look-back" strategies (used for recovering from a failure in the search tree); and "heuristics" (used for selecting the next literal to branch on).

- We considered CMOdels2 with various combinations of strategies, and other state-of-the-art systems like SMODELS, SMODELS $c c$, ASSAT, and DLV. 
- We conducted an extensive experimental analysis, involving all the above mentioned versions of CMODELS2 and systems, and a wide variety of tight and non tight programs, ranging from "small" randomly generated programs with a few hundred atoms, up to "large" programs with tens of thousands variables.

Our experimental results show that the look-back (resp. look-ahead) version of CMODELS2 has a clear edge over the other state-of-the-art systems that we considered on large (resp. small randomly generated) problems. The look-back version of CMODELS2 is very competitive also on the other non random, non large programs that we considered.

If we focus on the performances of the various versions of CMODELS2, the experimental results also point out that:

1. On the small randomly generated problems, "look-ahead solvers" (featuring a rather sophisticated look-ahead based on "failed literal", a simple look-back strategy - essentially backtracking- and a heuristic based on the information gleaned during the look-ahead phase) are best.

2. On the large problems, "look-back solvers" (featuring a simple but efficient look-ahead strategy -essentially unit-propagation with 2 literal watching-, a rather sophisticated look-back based on "learning" and a constant time heuristic based on the information gleaned during the look-back phase) are best.

3. Adding a powerful look-back (resp. look-ahead) to a look-ahead (resp. look-back) solver does not lead to better performances if the resulting solver is run on the small (resp. large) problems that we considered.

Using the terminology in (Giunchiglia et al., 2001), our comparison is "fair" because all the reasoning strategies are realized on a common platform and thus the experimental evaluation is not biased by the differences due to the quality of the implementation, and is "significant" because CMODELS2 implements current state-of-the-art lookahead/look-back strategies and heuristics. We believe that these results have important consequences both for developers and also for people interested in benchmarking ASP systems. For instance, our results say that we can hardly expect to develop one solver with the best performances on all the categories of problems. As a consequence,

- developers should focus on specific classes of benchmarks (e.g., on randomly generated programs), and 
- benchmarking should take into account whether solvers have been designed for specific classes of programs: Indeed, it hardly makes sense to run a solver designed for random (resp. large) programs on large (resp. random) programs.

The paper is structured as follows. In Section 2 we introduce the definitions, terminology and results at the basis of our work. Then, in Section 3 we present ASP-SAT in its basic backtracking version, and we prove its soundness and completeness. We also discuss in details what needs to be done in order to implement ASP-SAT on top of a SAT solver with learning. In Section 4 we show how we implemented ASPSAT in CMODELs. Section 5 contains the experimental, comparative evaluation. We end the paper with the conclusions and future work in Section 6.

A preliminary version of this paper is (Giunchiglia et al., 2004). This paper contains also results presented in (Giunchiglia and Maratea, 2005b; Giunchiglia and Maratea, 2005a).

\section{Formal Background}

\subsection{Syntax of Logid Programs}

A rule is an expression of the form

$$
p_{0} \leftarrow p_{1}, \ldots, p_{k}, \text { not } p_{k+1}, \ldots, \text { not } p_{m}, \text { not not } p_{m+1}, \ldots, \text { not } \operatorname{not} p_{n}
$$

$(0 \leq k \leq m \leq n)$ where $p_{0}$ is an atom or the symbol $\perp$ ( $\perp$ is the logical symbol standing for the empty disjunction, i.e., False), $p_{1}, p_{2}, \ldots, p_{n}$ are atoms, and the symbol not is the "negation" as failure operator. $p_{0}$ is the head of the rule, and the expression at the right of the arrow is the body. The intuitive meaning of a rule (1) is that $p_{0}$ is in the solution whenever the body is satisfied.

A (non disjunctive logic) program is a finite set of rules.

If the head of a rule is $\perp$, we call the rule a constraint. If a rule (1) contains an expression of the form not not $p_{i}$, then the rule is called nested, otherwise the rule is non nested or basic. If a logic program $\Pi$ contains at least one nested rule, $\Pi$ is a nested program, otherwise is non nested or basic. For instance, the program

$$
\begin{aligned}
& p \leftarrow \operatorname{not} \text { not } p \\
& q \leftarrow \text { not } p .
\end{aligned}
$$

is nested, while

$$
\begin{aligned}
& p \leftarrow p \\
& q \leftarrow \text { not } p .
\end{aligned}
$$


is non nested or basic.

\subsection{Answer Sets for Logic Programs}

In order to give the definition of an answer set we consider first the special case in which the program $\Pi$ does not contain the negation as failure operator not (i.e. for each rule (1) in $\Pi, n=m=k$ ). Let $\Pi$ be such a program and let $X$ be a set of atoms. We say that $X$ is closed under $\Pi$ if for every rule (1) in $\Pi, p_{0} \in X$ whenever $\left\{p_{1}, p_{2}, \ldots, p_{k}\right\} \subseteq X$. In the $n=m=k$ hypothesis, $\Pi$ has only one answer set, and it is the smallest set of atoms closed under $\Pi$. Computing such an answer set can be done in linear time, via the Dowling-Gallier procedure (Dowling and Gallier, 1984), or via unit-propagation (assuming the symbol " $\longleftarrow$ " is understood as the standard material implication, and "," as conjunction).

Now consider an arbitrary program $\Pi$. Let $X$ be a set of atoms. A rule

$$
p_{0} \leftarrow p_{1}, \ldots, p_{k}
$$

belongs to the reduct $\Pi^{X}$ of $\Pi$ with respect to $X$ if and only if there is a rule (1) in $\Pi$ with $X \cap\left\{p_{k+1}, \ldots, p_{m}\right\}=\emptyset$ and $\left\{p_{m+1}, \ldots, p_{n}\right\} \subseteq X$. $\Pi^{X}$ is a program without negation as failure. We say that a subset $X$ of the atoms in $\Pi$ is an answer set for $\Pi$ if $X$ is an answer set for $\Pi^{X}$ (Gelfond and Lifschitz, 1988; Lee and Lifschitz, 2003).

As an example, let $\Pi$ be the program (2) and consider the set of atoms $\{p\}$. The reduct $\Pi^{\{p\}}$ is

$$
p \leftarrow
$$

The set $\{p\}$ is the smallest set closed under (4) and hence it is also an answer set of the program $\Pi$. If we consider the set of atoms $\{p, q\}$, the reduct $\Pi^{\{p, q\}}$ is again (4). The set $\{p, q\}$ is not the smallest set closed under (4), and hence it is not an answer set of the program $\Pi$.

Determining the existence of an answer set for a program $\Pi$ is an $\mathrm{NP}$-complete problem. Indeed, checking if a set of atoms $X$ is an answer set of $\Pi$ can be done in linear time by first computing the reduct $\Pi^{X}$ and then computing the answer set of $\Pi^{X}$. NP-hardness can be easily proven using standard reductions of the SAT problem into logic programs under answer set semantics, see, e.g., (Janhunen, 2003).

\subsection{Completion}

Consider a program $\Pi$. For an atom $p_{0}$ the completion $\operatorname{Comp}\left(\Pi, p_{0}\right)$ of $\Pi$ relative to $p_{0}$ is the formula

$$
p_{0} \equiv \bigvee\left(p_{1} \wedge \ldots \wedge p_{k} \wedge \neg p_{k+1} \wedge \ldots \wedge \neg p_{m} \wedge p_{m+1} \wedge \ldots \wedge p_{n}\right)
$$


where the disjunction extends over all rules (1) in $\Pi$ with head $p_{0}$. The completion $\operatorname{Comp}(\Pi)$ of $\Pi$ consists of the formulas

$$
\bigvee_{i=1}^{k} \neg p_{i} \vee \bigvee_{i=k+1}^{m} p_{i} \vee \bigvee_{i=m+1}^{n} \neg p_{i}
$$

one for each rule (1) whose head is $\perp$; and of the formulas $\operatorname{Comp}\left(\Pi, p_{0}\right)$ for each atom $p_{0}$ in $\Pi$ (Clark, 1978; Lloyd and Topor, 1984). For instance, the completion of the program (2) consists of the formulas

$$
\begin{gathered}
p \equiv p \\
q \equiv \neg p,
\end{gathered}
$$

and (5) is also the completion of the program (3).

The following theorem, due to Marek and Subrahmanian (1989) for basic programs and generalized in (Erdem and Lifschitz, 2001) for nested programs, relates the answer sets of a program to the models of its completion. In the following, we say that a set of atoms $X$ satisfies (or is a model of) a set of formulas $\Gamma$ if $\Gamma$ is satisfied by the interpretation which assigns True to an atom $p$ if and only if $p \in X$.

THEOREM 1. Let $\Pi$ be a program. If $X$ is an answer set of $\Pi$, then $X$ satisfies the completion of $\Pi$.

The set of atoms $\{p, q\}$ does not satisfy the completion (5) of (2) (resp. (3)) and thus it is not an answer set of (2) (resp. (3)).

\subsection{Tight Programs}

Theorem 1 can be strengthened in the case of tight programs. A program $\Pi$ is tight if its dependency graph is acyclic. The dependency graph of a program $\Pi$ is the directed graph $G$ such that

- the nodes of $G$ are the atoms in $\Pi$, and

- for every rule (1) in $\Pi, G$ has an edge from $p_{0}$ to each atom in $\left\{p_{1}, \ldots, p_{k}\right\}$.

The following Theorem has been proved by Fages (1994) for basic programs, and it has been generalized by Erdem and Lifschitz (2001) to nested programs.

THEOREM 2. Let $\Pi$ be a tight program and $X$ a set of atoms. $X$ is an answer set for $\Pi$ iff $X$ satisfies the completion of $\Pi$. 
Program (2) is tight, while program (3) is non tight. Hence, according to the above theorem, the answer sets of (2) coincide with the models of (5) (and thus can be computed with SAT solvers).

\subsection{LoOp Formulas}

Theorem 1 states that if $X$ is an answer set of program $\Pi$ then $X$ satisfies $\operatorname{Comp}(\Pi)$. Theorem 2 says that the converse is also true if the program is tight. If the program is non tight, Lin and Zhao $(2002,2004)$ proved that to have the identity mapping between the answer sets of a basic program $\Pi$ and the models of its completion, we have to consider the loop formulas of $\Pi$. Lee and Lifschitz (2003) extended the concept of loop formulas to nested programs and proved that the same result holds with the extended definition. To formally state this last result, we need the following definitions.

A loop of $\Pi$ is a nonempty set $L$ of atoms such that for each pair $p, p^{\prime}$ of atoms in $L$ there exists a path of nonzero length from $p$ to $p^{\prime}$ in the dependency graph of $\Pi$ whose intermediate nodes belong to $L$.

Given a loop $L$, we define $R(L)$ to be the set of formulas

$$
\left(p_{1} \wedge \ldots \wedge p_{k} \wedge \neg p_{k+1} \wedge \ldots \wedge \neg p_{m} \wedge p_{m+1} \wedge \ldots \wedge p_{n}\right)
$$

for all rules (1) in $\Pi$, with $p_{0} \in L$ and $\left\{p_{1}, \ldots, p_{k}\right\} \cap L=\emptyset$. The loop formula associated with $L$ is

$$
\bigvee L \supset \bigvee R(L)
$$

where $\bigvee L$ denotes the disjunction of the atoms in $L$, and similarly for $\bigvee R(L)$.

THEOREM 3. Let $\Pi$ be a program. Let $\operatorname{Comp}(\Pi)$ be the completion of $\Pi$. Let $L F(\Pi)$ be the set of all the loop formulas associated with the loops of $\Pi$. For each set of atoms $X, X$ is an answer set of $\Pi$ iff $X$ is a model of $\operatorname{Comp}(\Pi) \cup L F(\Pi)$.

Consider the non tight program (3). Its completion is (5). The only loop of the program is $\{p\}$ and the loop formula associated with $\{p\}$ is

$$
p \supset \perp,
$$

which is equivalent to $\neg p$. Thus, the answer sets of (3) are the set of atoms that satisfy (5) and also $\neg p$. 


\section{SAT-based Answer Set Solvers}

\subsection{Previous approaches}

Cmodels (Lierler and Lifschitz, 2003) is an answer set solver based on SAT which has evolved along the years and which, in its current version, incorporates also the procedure described in this paper and in its predecessor (Giunchiglia et al., 2004). The version of CMODELS prior to (Giunchiglia et al., 2004) is restricted to tight programs, and, given a tight program $\Pi$, CMODELS

1. computes the completion $\operatorname{Comp}(\Pi)$ of the program, and

2. calls a SAT solver to find the models of $\operatorname{Comp}(\Pi)$ (corresponding to the answer sets of the input program). Before invoking the SAT solver, it may be necessary to convert the formulas in $\operatorname{Comp}(\Pi)$ to a set of clauses, as required by most SAT solvers. A clause is a disjunction of literals, and a literal is an atom or the negation of an atom.

The advantage of this method is that it uses SAT solvers as black boxes. On the other hand, it is restricted to tight programs.

Theorem 3 lays the foundation for extending this method to non tight programs.

Consider a program $\Pi$. To determine whether $\Pi$ has an answer set, one possibility is to

1. compute the completion and the loop formulas of $\Pi$, i.e., the set $\Gamma=\operatorname{Comp}(\Pi) \cup L F(\Pi)$ of formulas, and then

2. invoke a SAT solver to determine the models of (the clause conversion of the formulas in) $\Gamma$.

This is an "eager" ${ }^{1}$ approach which may work well in practice in some domains, but the resulting propositional formula may be exponentially bigger than the input program.

ASSAT (Lin and Zhao, 2002; Lin and Zhao, 2004) is a SAT-based system for basic programs which takes an alternative approach. Indeed, ASSAT adds loop formulas on demand, i.e., ASSAT

1. Computes $\Gamma=\operatorname{Comp}(\Pi)$.

1 The terminology is borrowed from the one used in decision procedures for separation logic, where "eager" approaches compile the input formula into an equisatisfiable propositional one, see, e.g., (Lahiri et al., 2002). 
2. Finds a model $X$ of $\Gamma$ by using a SAT solver (before this, it may be necessary to convert $\Gamma$ to a set of clauses). If no such model exists then the input program does not have answer sets and the procedure terminates returning False.

3. Checks if $X$ is an answer set: As we have already said in section 2.2, this can be done in linear time in the size of $\Pi$. If $X$ is an answer set, then the procedure terminates with returning True. Otherwise, ASSAT

a) finds at least one loop formula which is not satisfied by $X$, and adds it to $\Gamma$ : As described in section 4, also this step can be done in linear time in the size of $\Pi$; and

b) goes back to step 2 .

Lin and Zhao $(2002,2004)$ showed that ASSAT can often outperform rival systems. However, ASSAT has the following two drawbacks:

1. ASSAT is not guaranteed to work in polynomial space. Lifschitz and Razborov (2004) showed that there are programs $\Pi$ for which $L F(\Pi)$ contains exponentially many formulas (unless $P \nsubseteq N C^{1} /$ poly), each of which cannot be derived from the others and $\operatorname{Comp}(\Pi)$. For these programs $\Pi$ :

- If $\Pi$ has an answer set, then ASSAT performance on $\Pi$ depends on how lucky the system is in generating the right model first. In the best case it generates an answer set first. In the worst case it blows up in space.

- If $\Pi$ has no answer set, then ASSAT blows up in space. In fact, adding and keeping already added loop formulas is essential to guarantee that the SAT solver does not return an already computed model, and thus to guarantee ASSAT termination.

2. Considering two successive calls to the SAT solver, the computation done for finding the first model is completely discarded, i.e., not reused by the SAT solver in the second call. Thus some branches of the search tree may get computed many times.

Further considering the task of computing all answer sets of a program $\Pi$, there are two ways for doing it in ASSAT:

1. Compute $\operatorname{Comp}(\Pi) \cup L F(\Pi)$ and then call a SAT enumerator, i.e., a SAT solver able to return all the models of a propositional formula, e.g., MCHAFF (Moskewicz et al., 2001); or 
2. In order to avoid the generation of the same model $X$, once an answer set $X$ is found, modify ASSAT procedure in step 3 by

a) adding to $\Gamma$ one or more clauses ensuring that the same answer set $X$ is not re-computed, and

b) going back to step 2 .

For nested programs, the obvious clause to add to $\Gamma$ is

$$
\bigvee_{A \in X} \neg A \vee \bigvee_{A \notin X} A
$$

For basic programs, (i.e., of the kind that ASSAT considers) we can take advantage of the fact that the following anti-chain property holds: if $X$ is an answer set, no strict subset or superset of $X$ is an answer set. For these programs it is thus sufficient to add to $\Gamma$ one or both of the clauses

$$
\bigvee_{A \in X} \neg A, \quad \bigvee_{A \notin X} A
$$

in order to ensure that the same answer set is not re-computed. The advantage of adding (8) instead of (7) is that each clause in (8) entails (7) and thus it prunes more search space.

The first approach is unfeasible if there are (exponentially) many loop formulas. The second approach is unfeasible also when there are many answer sets.

\subsection{ASP-SAT WITH BACKTRACKING}

The above drawbacks can be eliminated if we do not use a SAT solver as a black-box. Instead, we can take advantage of that all the state-of-theart complete SAT solvers are based on the Davis-Logemann-Loveland procedure (Davis et al., 1962). The basic observation is that the DavisLogemann-Loveland procedure can easily work as a SAT enumerator.

Thus, given a program $\Pi$, we may first compute the completion of $\Pi$, and then

- generate the models of $\operatorname{Comp}(\Pi)$, and

- test whether the generated models are answer sets of $\Pi$.

We call ASP-SAT the resulting procedure, and it is represented -in its simple backtracking version - in Figure 1. In the figure, 


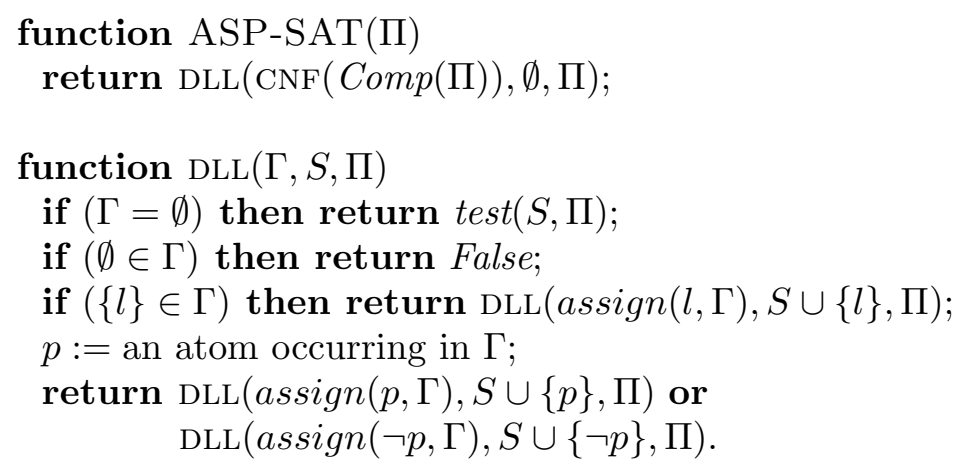

Figure 1. The SAT-based ASP-SAT procedure for Answer Set Programming

1. Given a set of formulas $\Gamma, \operatorname{CNF}(\Gamma)$ returns a set of clauses possibly with newly introduced propositional variables - such that, for any interpretation $\mu$ in the extended language, the following two properties hold:

a) if $\mu$ satisfies $\operatorname{CNF}(\Gamma)$ then the restriction of $\mu$ to the language of $\Gamma$ satisfies $\Gamma$, and

b) if $\mu$ satisfies $\Gamma$ then there exists an interpretation in the language of $\operatorname{CNF}(\Gamma)$ which $(i)$ extends $\mu$, and (ii) satisfies $\operatorname{CNF}(\Gamma)$.

An example of such a conversion is the "classical conversion" (which given a formula in negative normal form recursively distributes conjunctions over disjunctions), and the conversions based on "renaming", such as those described in (Tseitin, 1970; Plaisted and Greenbaum, 1986; Sheridan, 2004).

2. $l$ denotes a literal, and $\Gamma$ a set of clauses;

3. $S$ is an assignment, i.e., a consistent set of literals;

4. given an atom $p$, assign $(p, \Gamma)$ is the set of clauses obtained from $\Gamma$ by removing the clauses to which $p$ belongs, and by removing $\neg p$ from the other clauses in $\Gamma$. assign $(\neg p, \Gamma)$ is defined similarly.

A key feature of ASP-SAT is that it is based on DLL, which, considering its pseudo-code in the figure, is almost identical to the DavisLogemann-Loveland procedure: The only difference is that, when the empty set of clauses is generated, DLL invokes the function test $(S, \Pi)$ instead of just returning True. ASP-SAT thus follows a "lazy" approach to the computation of answer sets based on SAT, ${ }^{2}$ where, intuitively

\footnotetext{
2 The terminology is again borrowed from the one used in decision procedures for separation logic, where "lazy" approaches abstract the input formula into a
} 
speaking, the goal of the function test $(S, \Pi)$ is to return True if the assignment $S$ corresponds to at least one answer set of $\Pi$, and False otherwise. However, the function test $(S, \Pi)$ deserves some further comments. Assume $P$ is the set of atoms in the program $\Pi$. When the function $\operatorname{test}(S, \Pi)$ is invoked, its argument $S$ is such that $S \cap P$ satisfies the completion of $\Pi$ and is thus a candidate for being an answer set. However, it may be the case that $S$ is not a total assignment, i.e., it is possible that for some atom $p \in P$, neither $p$ nor $\neg p$ are in $S$. If $p$ is one such atom, also $(S \cap P) \cup\{p\}$ satisfies the completion of $\Pi$ and is thus another candidate for being an answer set. In general, an assignment $S$ can potentially correspond to exponentially many set of atoms satisfying the completion of $\Pi$, and each of them is a superset of the atoms in $S \cap P$. However, if $\Pi$ is a basic program, none of these strict supersets is an answer set of $\Pi$, as established by the following proposition.

PROPOSITION 4. Let $\Pi$ be a basic program. Let $X$ be a set of atoms satisfying Comp( $\Pi)$. If $X \subset X^{\prime}$ then $X^{\prime}$ is not an answer set of $\Pi$.

Proof. We are given that $X$ satisfies $\operatorname{Comp}(\Pi)$. From completion construction, it follows that $X$ is closed under $\Pi^{X}$. Since $X \subset X^{\prime}$ and $\Pi$ is basic, $\Pi^{X^{\prime}} \subseteq \Pi^{X}$. Hence $X$ is closed under $\Pi^{X^{\prime}}$, and thus $X^{\prime}$ is not the smallest set closed under $\Pi^{X^{\prime}}$.

Thus, according to the above proposition, if $\Pi$ is tight, $\operatorname{test}(S, \Pi)$ has just to check if $S \cap P$ is an answer set of $\Pi$ : Any set of atoms extending $S \cap P$ is not an answer set.

We are now ready to state our main Theorem in the case of basic programs.

THEOREM 5 (Soundness and completeness for basic programs). Let $\Pi$ be a basic program in the set $P$ of atoms. Let test $(S, \Pi)$ be a function returning True if $S \cap P$ is an answer set of $\Pi$, and False otherwise. ASP-SAT $(\Pi)$ returns True if $\Pi$ has an answer set, and False otherwise.

propositional one and refine the propositional model if it does not correspond to a model of the original formula, see, e.g., (Armando et al., 1999; de Moura et al., 2002; Barrett et al., 2002; Armando et al., 2005). More recently (Nieuwenhuis and Oliveras, 2005) showed that better performances can be obtained by using a lazy approach in which the assignment is extended on the basis of the semantics of the original formula in separatin logic. In our setting, this would correspond to assign some atoms - not entailed by the current assignment and the completion of the input program - but entailed by the current assignment, the completion of the input program and the set of loop formulas: Whether this can lead to better performances is still an open research issue. 
Proof. Soundness is trivial. For completeness, assume that ASPSAT(П) returns False. Let $P$ be the set of atoms in $\Pi$. Let $\Gamma$ be the set of assignments $S$ that have been checked, i.e., such that test $(S, \Pi)$ has been invoked. The fact that $\Pi$ has no answer sets follows from the following properties

1. The formula

$$
\bigvee_{S \in \Gamma}\left(\bigwedge_{p: p \in S, p \in P} p \wedge \bigwedge_{p: \neg p \in S, p \in P} \neg p\right)
$$

is logically equivalent to the completion $\operatorname{Comp}(\Pi)$ of $\Pi$ (Proposition 5 in (Giunchiglia et al., 2002), restated as Lemma 4 in (Armando et al., 2005)).

2. The set of answer sets of $\Pi$ is a subset of $\{S \cap P: S \in \Gamma\}$ (easy consequence of Theorem 1 and Proposition 4).

Proposition 4 does not hold for arbitrary programs. In general, given a nested program $\Pi$, it is possible that two sets $X$ and $X^{\prime}$ of atoms are such that

- $\quad X$ satisfies the completion of $\Pi$ but is not an answer set of $\Pi$, and

- $\quad X^{\prime}$ is a superset of $X$ and is an answer set of $\Pi$.

This is illustrated by the following program:

$$
\begin{aligned}
& p_{1} \leftarrow \text { not not } p_{1} \\
& p_{2} \leftarrow p_{1} \\
& p_{2} \leftarrow p_{2} .
\end{aligned}
$$

The completion of the program is $\left\{p_{1} \equiv p_{1}, p_{2} \equiv\left(p_{1} \vee p_{2}\right\}\right.$. The set of atoms $\left\{p_{2}\right\}$ satisfies the completion but is not answer set. The set of atoms $\left\{p_{1}, p_{2}\right\}$ is a superset of $\left\{p_{2}\right\}$ and is also an answer set of (9).

Thus, in the general case, whenever test $(S, \Pi)$ is invoked, every set $X$ of atoms which is

1. a superset of $S \cap P$, and

2. a subset of $\{p: \neg p \notin S, p \in P\}$

has to be checked to see if it is an answer set of $\Pi$.

THEOREM 6 (Soundness and completeness for arbitrary programs). Let $\Pi$ be a program in the atoms $P$. Let test $(S, \Pi)$ be a function returning True if there exists a set $X$ with $S \cap P \subseteq X \subseteq\{p: \neg p \notin S, p \in P\}$ which is an answer set of $\Pi$, and False otherwise. ASP-SAT( $\Pi)$ returns True if $\Pi$ has an answer set, and False otherwise. 
Proof. The proof is analogous to the one of Theorem 5, the only difference is that, assuming

- $P$ is the set of atoms in $\Pi$,

- $\Gamma$ is the set of assignments $S$ that have been checked, i.e., such that $\operatorname{test}(S, \Pi)$ has been invoked,

the set of answer sets of $\Pi$ is a subset of

$$
\{X: \exists S \in \Gamma . S \cap P \subseteq X \subseteq\{p: \neg p \notin S, p \in P\}\},
$$

as established by Theorem 1 .

\subsection{ASP-SAT WITH LEARNING}

The ASP-SAT procedure in the previous subsection is based on the standard Davis-Logemann-Loveland procedure with simple chronological backtracking. It is thus not infrequent for ASP-SAT to explore a possibly large subtree whose leaves are all dead-ends because of some bad choices performed way up in the search tree. In SAT, the standard solution to this problem is to backjump over the choices that do not belong to the "reason" for the failure. Intuitively, if $S$ is an assignment which falsifies the input set $\Gamma$ of clauses, then a reason $R$ for $S$ is a subset of the literals in $S$ such that any assignment extending $R$ falsifies $\Gamma$. (We say that a set $S$ of literals falsifies a set of formulas $\Gamma$ if $S \cup \Gamma$ is inconsistent). Reasons are initialized as soon as a failure is generated, and updated while backtracking. Many of the current state-of-the-art SAT procedures feature such backjumping mechanism and extend it with learning: under certain conditions, a reason $R$ is converted into the clause $\left(\bigvee_{p \in R} \neg p \vee \bigvee_{\neg p \in R} p\right)$ which is then learned, i.e., added to the input set of clauses as additional constraint. Since exponentially many distinct reasons can be computed, suitable criteria are also used in order to forget (i.e., remove) clauses corresponding to reasons, thus maintaining the SAT solver in polynomial space.

It is out of the goals of this paper to describe how learning is incorporated in the Davis-Logemann-Loveland procedure: See, e.g., (Dixon et al., 2004) for a high-level description of learning including soundness and completeness statements of the resulting procedure, (Silva and Sakallah, 1996; Bayardo, Jr. and Schrag, 1997; Zhang et al., 2001) for more detailed descriptions of different learning mechanisms. For our purposes, it suffices to say that a SAT solver with learning can still be used as underlying procedure for ASP-SAT. The only difference with respect to the procedure in Figure 1 is in the test procedure. In fact, 
as we outlined above, whenever we have a failure we have to have also a corresponding reason. In our case, if test $(S, \Pi)$ returns False, it has also to return a subset $R$ of the atoms in $S$ such that for any total assignment $S^{\prime}$ extending $R$ and not falsifying the completion of $\Pi$, the set of atoms in $S^{\prime}$ is guaranteed to be not an answer set of $\Pi$. One such set $R$ is $S$. However, in order to maximize the effects of the backjumping and learning mechanisms in the SAT solver, it is important that $R$ be as small as possible. In the case of a basic program, one smaller such set is the set of atoms in $S$ (see Proposition 4). However, it is possible to take advantage of loop formulas, and - in practice - return reasons which are often less than $1 \%$ of the size of $S$.

To illustrate how loop formulas can help for computing small reasons, consider a call to test $(S, \Pi)$, and let $P$ be the set of atoms in $\Pi$. We assume that $S$ does not correspond to any answer set of $\Pi$, otherwise test $(S, \Pi)$ has just to return True and the computation of a reason does not make sense.

For simplicity, assume that $S$ is a total assignment. The idea is to find a loop formula $F$ which is falsified by $S$, and return a subset $S^{\prime}$ of $S$ necessary to falsify $F$ : Since every answer set of $\Pi$ has to satisfy all the loop formulas of $\Pi$, the set of atoms in any superset of $S^{\prime}$ is guaranteed to be not an answer set of $\Pi$. Important is the fact that determining such a set $S^{\prime}$ can be done efficiently, i.e., in linear time in the size of $\Pi$, as detailed in the next section.

If $S$ is not total but $\Pi$ is basic, then - thanks to Proposition 4-we can just consider the total assignment $S \cup\{\neg p: p \in P, p \notin S\}$.

Now assume that $\Pi$ is nested and that $S$ is not total. Assume for simplicity that there is only one atom $p \in P$ such that neither $p$ nor $\neg p$ is in $S$. Let $S_{1}=S \cup\{p\}$ and $S_{2}=S \cup\{\neg p\}$. Both $S_{1}$ and $S_{2}$ are total. Furthermore, $S_{1} \cap P$ and $S_{2} \cap P$ are not answer sets, and we can compute $S_{1}^{\prime} \subseteq S_{1}$ and $S_{2}^{\prime} \subseteq S_{2}$, each falsifying a loop formula of $\Pi$ as in the previous case. If $p \notin S_{1}^{\prime}$ (resp. $\neg p \notin S_{2}^{\prime}$ ) then $S_{1}^{\prime}$ (resp. $S_{2}^{\prime}$ ) is also a subset of $S$ and can be returned. If $p \in S_{1}^{\prime}$ and $\neg p \in S_{2}^{\prime}$ we can safely return $S^{\prime \prime}=S_{1}^{\prime} \cup S_{2}^{\prime} \backslash\{p, \neg p\}: S^{\prime \prime} \subseteq S$ and no set extending $S^{\prime \prime}$ can correspond to an answer set. The above procedure can be easily extended to the case in which there are more than one atoms $p \in P$ with $\{p, \neg p\} \cap S=\emptyset$.

Notice that $S$ may be a non total assignment because in ASP-SAT test $(S, \Pi)$ is invoked whenever the input set of clauses is empty. Indeed, many SAT solvers - including MCHAFF - have a different termination condition for True: True is returned whenever either $p$ or $\neg p$ is in $S$, for each atom $p$ in the input set of clauses $\Gamma$. Assuming that all the atoms in $\Pi$ occur also in $\Gamma$, the above termination condition for True ensures that $S$ is total. 
We want to remark that in order to guarantee the termination of our procedure ASP-SAT( $\Pi)$, it is not necessary to store the reasons returned by test $(S, \Pi)$ : On the other hand, learning (a polynomial amount of) reasons can improve performances of the procedure. Consider in fact the program $\Pi_{k}$ consisting of the rules

$$
p_{i} \leftarrow p_{i+1} \quad p_{i+1} \leftarrow p_{i}
$$

where $i \in\{0,2, \ldots, 2 k-2\}$, and of the constraint

$$
\perp \leftarrow \text { not } p_{0}, \text { not } p_{1}, \ldots, \text { not } p_{2 k-1} .
$$

$\Pi_{k}$ has no answer set, while $\operatorname{Comp}\left(\Pi_{k}\right)$ has $2^{k}-1$ models. Assuming $\operatorname{CNF}\left(\operatorname{Comp}\left(\Pi_{k}\right)\right)$ consists of the clauses

$$
\neg p_{i} \vee p_{i+1} \quad \neg p_{i+1} \vee p_{i}
$$

$(i \in\{0,2, \ldots, 2 k-2\})$, and

$$
p_{0} \vee p_{1} \vee \ldots \vee p_{2 k-1},
$$

the following facts hold (in this paragraph, for simplicity, we assume that the clauses corresponding to the reasons returned by $\operatorname{test}\left(S, \Pi_{k}\right)$ are learned and never forgotten):

- A naive implementation of $\operatorname{test}\left(S, \Pi_{k}\right)$ which returns $S$ as reason for its failure, will cause the generation and rejection of exponentially many sets of atoms, one for each set of atoms satisfying the completion of $\Pi_{k}$;

- Since $\Pi_{k}$ is basic, test $\left(S, \Pi_{k}\right)$ may return the set of atoms in $S$ as reason for its failure. Depending on the order in which the assignments are generated and then tested, different things can happen, ranging in between the following two extreme cases:

1. In the best case, the assignments containing exactly one pair $\left\{p_{i}, p_{i+i}\right\}$ ( $i$ even) are generated (and then rejected) first: In this case, the clause $\left(\neg p_{i} \vee \neg p_{i+1}\right)$ is learned, and, together with (10), this implies that any other assignment generated afterwards will contain both $\neg p_{i}$ and $\neg p_{i+1}$. After the $k$ sets with two positive atoms are generated, the resulting set of clauses is inconsistent and no more assignments are generated.

2. In the worst case, the assignments containing a maximum number of positive atoms in $P$ are generated (and then rejected) first: The first assignment that will be generated is $\left\{p_{0}, p_{1}, \ldots, p_{2 k-1}\right\}$, and the corresponding learned clause is $\neg p_{0} \vee$ 
$\neg p_{1} \vee \ldots \vee \neg p_{2 k-1}$, and it is easy to see that exponentially many assignments will be generated before determining the non existence of answer sets.

- An implementation of test $(S, \Pi)$ that returns a subset of $S$ falsifying one of the loop formulas is guaranteed to test $k$ assignments. This is due to the fact that $\Pi_{k}$ has $k$ loops, $\left\{p_{i}, p_{i+1}\right\}$, with $i$ even. Given a loop $\left\{p_{i}, p_{i+1}\right\}$, its loop formula is $\left(p_{i} \vee p_{i+1}\right) \supset \perp$, corresponding to

$$
\left(\neg p_{i} \wedge \neg p_{i+1}\right) .
$$

Given a call to test $(S, \Pi),(i)$ a loop formula of the form (11) falsified by $S$ is computed; ( $i i)$ the two possible subsets of $S$ falsifying (11) are computed, i.e., $\left\{p_{i}\right\}$ and $\left\{p_{i+1}\right\}$; (iii) one of them is returned as reason; (iv) assuming $\left\{p_{i}\right\}$ is the returned reason, the clause $\left\{\neg p_{i}\right\}$ is learned; and $(v)$ after backtracking/backjumping, unit-propagation immediately assigns both $p_{i}$ and $p_{i+1}$ to False.

After $k$ calls to the test $(S, \Pi)$ procedure, the resulting set of clauses is unsatisfiable.

\subsection{Computational properties of ASP-SAT}

From a computational perspective, the ASP-SAT procedure in Figure 1 has the following features:

1. It performs the search on $\operatorname{Comp}(\Pi)$ and thus does not introduce any extra variables except for those possibly needed by the clause form transformation.

2. It is guaranteed to work in polynomial space.

3. It can deal with both tight and non tight programs: In the case of tight problems, for each call to test $(S, \Pi)$, the set of atoms of $\Pi$ which are also in $S$, is guaranteed to be an answer set of $\Pi$, and thus ASP-SAT behaves as a standard SAT solver running on $\operatorname{CNF}(\operatorname{Comp}(\Pi))$.

If the underlying SAT solver uses learning, then all the above features still hold (assuming that the SAT solver itself works in polynomial space).

Compared to the version of CMODELS prior to (Giunchiglia et al., 2004), ASP-SAT is not restricted to work on tight programs.

Compared to ASSAT, ASP-SAT is guaranteed to work in polynomial space and has also the following advantages: 
- It is easily modifiable to return all the answer sets: Assuming the solver is based on backtracking, the only thing that is needed is to modify $\operatorname{test}(S, \Pi)$ in order to

1. print the set of atoms determined to be an answer set, and

2. return False.

Assuming the solver is based on learning, test $(S, \Pi)$ has to

1. print the set of atoms determined to be answer sets, and

2. return False and a reason $R \subseteq S$ such that each assignment extending $R$ corresponds to already computed answer sets. If $\Pi$ is a basic program then the anti-chain property holds for $\Pi$ : As a consequence, the set of atoms in $S \cap P$ is one such a reason, and the subset of $S$ consisting of the negation of the atoms in $P$ is another possibility. If $\Pi$ is a nested program, the set $S$ itself has to be returned.

- No computation is ever repeated. When test $(S, \Pi)$ fails, instead of restarting the search from scratch as done in ASSAT, the computation is restarted from the same point in the search tree where test $(S, \Pi)$ was called: the search then continues from this point following the depth-first search schema of the algorithm.

On the other hand, ASSAT advantage over ASP-SAT is that the SAT solver is used as a black-box without any need of even minor modifications.

Compared to other state-of-the-art answer set solvers like SMODELS, SMODELS $_{c c}$ and DLV, ASP-SAT has the advantage of being SAT-based, and thus it can leverage on the great amount of knowledge available in SAT. For instance, we are not aware of any non SAT-based answer set solver using the analogous of two-literal watching data structures for efficiently pruning the search tree while descending it.

\section{Implementation in Cmodels}

\subsection{Integration in CMOdels}

We have integrated our implementation of ASP-SAT in CMODELS. CMODELS2 is the name that we use for the resulting system.

The input language of CMODELS2 is a grounded logic program that can be generated by the front-end LPARSE (Syrjanen, 2003), and is the same as the input language of SMODELS, SMODELS ${ }_{c c}$ and ASSAT. The 
input may thus contain basic rules as well as choice, cardinality and weight constraint rules (Syrjanen, 2003, Sections 5.3, 5.4). A choice rule has the form

$$
\left\{p_{01}, \ldots, p_{0 j}\right\} \leftarrow p_{1}, \ldots, p_{k}, \text { not } p_{k+1}, \ldots, \text { not } p_{m}
$$

where each $p$ with a subscript is an atom. The intuitive meaning of a choice rule is that any atom contained in $\left\{p_{01}, \ldots, p_{0 j}\right\}$ may or may not belong to the solution whenever the body is satisfied. A weight constraint rule is an expression of the form

$$
p_{0} \leftarrow L\left\{p_{1}=w_{1}, \ldots, p_{k}=w_{k}, \text { not } p_{k+1}=w_{k+1}, \ldots, \text { not } p_{m}=w_{m}\right\} W
$$

where $L, U, w_{1}, \ldots w_{m}$ are integers, and each $p_{i}(i=0, \ldots, m)$ is an atom. The intuitive meaning of such rule is that $p_{0}$ is in the solution if the sum of the weights of the satisfied literals in the body of the rule is between $L$ and $U$. A cardinality constraint rule is a weight constraint rule in which all the integers in $\left\{w_{1}, \ldots, w_{m}\right\}$ are equal to 1 .

It is out of the scope of this paper to describe the semantics of programs with these rules in details, see, e.g., (Simons et al., 2002). For our goals, it is sufficient to say that in CMODELS2 weight constraint and choice rules are eliminated by introducing auxiliary atoms and nested rules as described in (Lifschitz et al., 1999; Ferraris and Lifschitz, 2005).

Traditionally, CMODELS was restricted to find answer sets for tight programs, via the following steps (see (Lierler and Lifschitz, 2003) for more details):

1. Simplification of the input LPARSE program, performing operations similar to those involved in SMODELS.

2. Elimination of choice and weight constraints rules in favor of nested rules.

3. Verification that the resulting program (possibly with nested rules) is tight.

4. Construction of the program's completion, conversion to a set of clauses, and call to a SAT solver. The clause conversion takes linear time and introduces up to $m$ new atoms, where $m$ is the number of rules in the program.

In CModels2, step 3 is not needed anymore (and is no longer performed) since a tight program can be considered as a particular case of a non tight one in which each call to test $(S, \Pi)$ succeeds. 


\subsection{ASP-SAT IMPLEMENTATION}

ASP-SAT is implemented on top of the SIMO system (Giunchiglia et al., 2003). SIMO is a MCHAFF-like SAT solver and thus features unit-propagation based on a two-literal watching data structure, 1UIP learning and VSIDS heuristics (see (Moskewicz et al., 2001) for a description of these techniques). However, it does not feature the low level optimizations of MCHAFF, and thus it is on average within a factor of 3 slower than MCHAFF. We have used SIMO because is the system we know better, and this allowed us to a relatively easy integration of the other search strategies and heuristics used for the experimental analysis.

With reference to Figure 1, in order to use SIMO as a search engine in an ASP solver, we had to modify it in order to

1. call test $(S, \Pi)$ whenever True was returned, and

2. guarantee that each set $S$ of literals in $\operatorname{test}(S, \Pi)$ is total.

Considering the second task, SIMO - like all the MCHAFF-based SAT solvers - returns True when all the atoms in the input set of clauses are assigned and no empty clause has been generated. However, SIMO input set of clauses may not contain all the atoms in the input program. Indeed, as a preliminary step and before the search starts, SIMO (and many other SAT solvers as well) pre-processes the input set of clauses and

1. eliminates tautological clauses (i.e., clauses with both an atom and its negation as disjuncts),

2. assigns pure literals, i.e., each atom $p$ is assigned to True if $\neg p$ does not belong to any clause in the input formula, and similarly for $\neg p$.

These operations are not harmful in SAT solving. However, if the SAT solver is used - as in our case - as basis for an answer set solver, both operations may lead to incorrect results. Consider in fact the program

$$
\begin{aligned}
& p_{1} \leftarrow \text { not not } p_{1} \\
& p_{2} \leftarrow p_{1} \\
& p_{2} \leftarrow p_{2} \\
& \perp \leftarrow \text { not } p_{1}, \text { not } p_{2}
\end{aligned}
$$

which has $\left\{p_{1}, p_{2}\right\}$ as answer set. The completion of the program is $\left\{p_{1} \equiv p_{1}, p_{2} \equiv\left(p_{1} \vee p_{2}\right), p_{1} \vee p_{2}\right\}$. Considering the straightforward translation to a set of clauses, and after the elimination of the tautological clauses, 
1. only two clauses are left, i.e., $\left(\neg p_{1} \vee p_{2}\right),\left(p_{1} \vee p_{2}\right)$, and

2. after $p_{2}$ is assigned during the pre-processing, the empty set of clauses is generated.

The empty assignment is returned and is checked to see if it is an answer set. Since it is not, False would be incorrectly returned. In order to avoid such undesired behavior, sIMO pre-processing has been modified in order to keep tautological clauses, and to not assign pure literals.

In order to evaluate the impact of different search strategies and heuristics in solving answer set programs, we have enhanced SIMO with search strategies and heuristics other than those implemented by MCHAFF. In particular, we implemented:

- Failed-literal detection: before branching, for each unassigned atom $p, p$ is assigned to True and then unit-propagation is called again: If a contradiction is found, $p$ is said to be a failed literal, $\neg p$ can be safely assigned, and unit-propagation is again performed. Otherwise, $\neg p$ is checked following the same procedure implemented for $p$.

- Standard backtracking: learning is disabled, and recovery from failure is performed by chronologically backtracking to the latest assigned branching literal.

- The unit heuristic, based on the failed-literal detection technique. Given an unassigned atom $p$, while doing failed-literal on $p$ we count the number $u(p)$ of unit-propagation caused, and then we select the atom with maximum $1024 \times u(p) \times u(\neg p)+u(p)+u(\neg p)$. The atom is assigned to True first.

The above search strategies and heuristics are not novel: they are standard techniques in the SAT field, and are implemented by many state-of-the-art SAT solvers. Indeed, current state-of-the-art SAT solvers can be divided in two main categories:

- "look-ahead" solvers, featuring a rather sophisticated look-ahead based on "failed literal", a simple look-back (essentially backtracking) and a heuristic based on the information gleaned during the look-ahead phase. These solvers are best for dealing with "small but relatively difficult" randomly generated $k$-cnf formulas. A solver in this category is SATZ ( $\mathrm{Li}$ and Anbulagan, 1997).

- "look-back" solvers, featuring a simple but efficient look-ahead (essentially unit-propagation with 2 literal watching), a rather sophisticated look-back based on "1-UIP learning" and a constant time 
heuristic based on the information gleaned during the look-back phase. These solvers are best for dealing with "large but relatively easy" instances, typically encoding non random problems. A solver in this category is MCHAFF (Moskewicz et al., 2001).

3 By combining SIMO original reasoning strategies with those newly implemented, we can obtain both a MCHAFF-like and a SATZ-like SAT solver, and consequently, a "look-back" answer set solver, and a "lookahead" answer set solver. Our goal is to confirm the expectations that

- on randomly generated problems, look-ahead solvers are best, while

- on large problems, look-back solvers are best

also in answer set programming. Given that all the different search strategies are implemented, combined and analyzed in a common platform, our results are not biased by differences in the quality of the underlying implementations.

\subsection{Implementation of test $(S, \Pi)$}

Consider a call to test $(S, \Pi)$, i.e., such that $S$ is a total assignment not falsifying the completion of $\Pi$. Let $X$ be the set of atoms in $S$ and in $\Pi$.

The primary goal of $\operatorname{test}(S, \Pi)$ is

1. to verify if $X$ is an answer set of $\Pi$, and

2. to compute a subset $R$ of $S$ to be used as reason if the SAT solver uses learning.

In our implementation, the computation of the reason involves looking for a loop formula of $\Pi$ which is falsified by $S$. To describe the procedure, the following terminology will be used: In a graph, a loop $L$ is maximal if it is a strongly connected component, and is also terminating (using stardard definition) if there is no other maximal loop $L^{\prime}$ with a path from $L$ to $L^{\prime}$.

Assuming learning is enabled, test $(S, \Pi)$ consists of the following steps:

1. Compute the reduct $\Pi^{X}$ of $\Pi$ with respect to $X$;

\footnotetext{
3 The terminology "small but relatively difficult" and "large but relatively easy" refer to the number of atoms and are used to convey the basic intuitions about the instances. To get a more precise idea in SAT, consider that in the SAT2003 competition, instances in the random and industrial categories had, on average, 442 and 42703 atoms respectively (Le Berre and Simon, 2003).
} 
2. Compute the answer set $X^{\prime}$ of $\Pi^{X}$ in linear time via the DowlingGallier procedure (Dowling and Gallier, 1984);

3. If $S^{\prime}=X \backslash X^{\prime}$ is empty then return True: $X$ is an answer set of $\Pi$ ( $X^{\prime}$ is by construction guaranteed to be a subset of $X$ ). Otherwise,

4. Considering the dependency graph of $\Pi$ restricted to the nodes in $S^{\prime}$, a terminating maximal loop $L$ is computed, and the corresponding loop formula $F$ is determined. $X$ does not satisfy $F$ : This result has been established in (Lin and Zhao, 2002) for basic programs, and it has been generalized to include nested programs in (Lierler, 2005).

5. $F$ has the form (6) and since $X$ is a superset of $L, X$ does not satisfy each of the formulas in $R(L)$. Since each formula $G$ in $R(L)$ is a conjunction of literals, $G$ is traversed looking for a literal whose complementary belongs to $S$. This literal is added to the returned reason and the whole procedure is iterated till all the formulas in $R(L)$ are analyzed.

Each of the above steps takes at most linear time in the size of the program. The above described procedure for computing a maximal terminating loop falsified by $S$ is the same as the one described in (Lin and Zhao, 2004), generalized to handle also nested programs. The key difference between our approach and Lin and Zhao's is that they add the loop formula to the input set of clauses and then call again the SAT solver from scratch. Here, the loop formula is only used to find a (small) subset of $S$ to be used as reason: As we already said, our procedure is guaranteed to be sound, complete and working in polynomial space even assuming the entire set $S$ is returned (thus, without making any use of loop formulas).

If learning is disabled (as in CMODELS2 version with backtracking), step 3 in the above description of $\operatorname{test}(S, \Pi)$ is modified in order to return True if $X \backslash X^{\prime}$ is empty, and False otherwise.

\section{Experimental Results}

\subsection{Solvers, BENCHMARKS AND SETTING}

In order to evaluate the effectiveness of our approach, we comparatively tested CMODELS2 against other state-of-the-art systems on a variety of benchmarks. The systems we considered are SMODELS version 2.27, 
SMODELS $_{c c}$ version 1.08, ASSAT version 2.00, DLV release of 2005-02$23 .{ }^{4}$ It worths remarking that while SMODELS, SMODELS $c c$, ASSAT and CMOdels2 use LPARSE as preprocessor, and thus can be run on the same input files, DLV does not. This explains why DLV has been run only on a few benchmarks. Analogously, ASSAT can only deal with basic programs and thus it has not been run on some instances. Finally, for DLV we mention that it is a system specifically designed for disjunctive logic programs, and that very different results can be obtained depending on the specific encoding being used.

Considering CMODELS2, we have the possibility to combine different look-ahead/look-back search strategies and heuristics. In order to keep track of which combination we are using, we will refer to a combination of search strategies and heuristics using an acronym where the first, second and third letter denote the look-ahead, look-back and heuristic used, respectively. We considered 4 combination of reasoning strategies:

1. ulv: our default answer set solver, incorporating a MCHAFF-like lookback SAT solver, with standard Unit propagation, backtracking enhanced with Learning, and VSIDS heuristic.

2. fbu: a standard SATZ-like look-ahead solver, with unit propagation enhanced with Failed literal detection, standard Backtracking, and the Unit heuristic.

3. flv: an hybrid solver, featuring unit propagation enhanced with Failed literal detection, backtracking enhanced with Learning, and the VSIDS heuristic.

4. flu: another hybrid solver, featuring unit propagation enhanced with Failed literal detection, backtracking enhanced with Learning, and the Unit heuristic.

We considered only these 4 combinations of reasoning strategies and heuristics because, besides of being the most significant, the other possible combinations do not make even sense: VSIDS heuristic requires "learning" in order to be significant, while unit heuristic requires failedliteral. fbu and ulv are the two solvers that we expect to perform best on randomly generated programs and on large programs respectively. Assuming that the expectations are met, the performances of the two hybrid solvers are of interest in order to

\footnotetext{
${ }^{4}$ See http://www.tcs.hut.fi/Software/smodels/, http://www.nku.

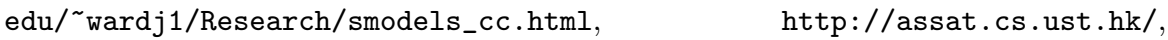
http://www.dbai.tuwien.ac.at/proj/dlv/
} 
- determine whether adding a powerful look-back (resp. look-ahead) to a look-ahead (resp. look-back) solver leads to better performances on randomly generated (resp. large) programs.

- get indications about which combination of reasoning strategy is the most promising on non randomly generated and non large programs.

All the solvers where run in their plain (optimal) configuration unless suggested by the authors. For examples, SMODELS ${ }_{c c}$ has been run with option "-nolookahead" (look-ahead turned off) as explicitly suggested by the authors in the SMODELS $c c$ 's home page. For ASSAT, we had to increase its internal limit on the number of atoms in the (grounded) logic program (variable C_MAXATOM).

About the benchmarks, our test-set includes both tight and non tight, both randomly generated and non randomly generated programs. Each benchmark belongs to a class of publicly available programs which have been used before in the literature, or to a class of benchmarks for which a generator is available. In this last case, we may have generated bigger instances than those reported in the literature. In order to validate our expectations, we divide the benchmarks in three categories, being $(i)$ randomly generated programs, $(i i)$ "large" programs with more than (approximately) 10000 atoms, and (iii) other problems not falling in the previous categories. We say that a program is basic when each rule has the form (1) where $n=m$, and non basic when a program contains choice rules or weight constraints. Recall that choice and weight constraint rules are eliminated with the help of auxilary atoms and nested rules of the form (1).

The results of the solvers on the most difficult instances of each class is given by means of tables, as it is customary in the answer set literature. In the tables,

1. The first column is a progressive number.

2. The second column is the ratio between number of rules and number of atoms for random problems, and the name of the benchmark in case it is a non randomly generated program.

3. The third column contains the number of atoms (\#VAR) after grounding. For non random problems, a "+" to the left of the name indicates that the instance has answer sets.

4. The remaining columns are one per solver, and they indicate its performances. 
For each row, the best result is in bold, and the results within a factor of 2 from the best are underlined.

Finally, all the tests were run on a Pentium IV PC, with $2.8 \mathrm{GHz}$ processor, 1024MB RAM, running Linux. For SMODELS, SMODELS $c$, ASSAT and CMODELS2, the time taken by LPARSE is not counted. ${ }^{5}$ Further, each system was stopped after 3600 seconds of CPU time on non random problems, and 600 seconds on random problems, or when it exceeded all the available memory. In the tables, these cases are denoted with "TIME" and "MEM" respectively. Otherwise, the tables report the CPU times in seconds needed by each solver to solve the problem. Some of the results here presented have also been presented in (Giunchiglia et al., 2004; Giunchiglia and Maratea, 2005b; Giunchiglia and Maratea, 2005a): All the experiments have been relaunched. This justifies the minor differences in the results, especially with (Giunchiglia et al., 2004), where the experiments were condutected on a Pentium IV PC, with $1.8 \mathrm{GHz}$ processor, 512MB RAM DDR 266MHz, running Linux.

\subsection{RANDOMLY GENERATED PROGRAMS}

Table I shows the results for "small" programs, randomly generated according to two different methodologies:

1. Problems (1)-(10) are translation of randomly generated $k$-SAT instances. A $k$-SAT instance consists of $L$ distinct clauses, where each clause is generated by randomly selecting $k$ different atoms and negating each with probability 0.5. The number of distinct possible atoms in a $k$-SAT instance is a priori fixed and denoted with $N$. Then, each $k$-SAT instance $F$ is converted to a program as follows

- if $C=\left(l_{1} \vee \ldots \vee l_{k}\right)$, we define $\operatorname{sat2tlp}(C)$ to be the rule $\perp \leftarrow$ not $l_{1}, \ldots$, not $l_{k}$ where not $l$ is $p$ if $l=\neg p$ and is not $p$ if $l$ is the atom $p$;

- Then, if $F$ is a $k$-SAT instance, the translation of $F$, is

$$
\cup_{C \in F} \operatorname{sat2tlp}(C) \cup \cup_{p \in P}\left\{p \leftarrow \text { not } p^{\prime}, p^{\prime} \leftarrow \text { not } p\right\}
$$

where, for each atom $p \in P, p^{\prime}$ is a new atom associated to $p$. These benchmarks are tight, and have been used in (Faber et al., 2001; Simons et al., 2002; Ward and Schlipf, 2004).

\footnotetext{
${ }^{5}$ Adding the times of LPARSE would not change the picture for DLV when compared to CMODELS2 and other systems.
} 
Table I. Performances on randomly generated logic programs. Problems (1)-(10) are tight programs being the translation of 3-SAT benchmarks. Problems (11)-(20) are randomly generated logic programs using Lin and Zhao's methodology.

\begin{tabular}{|c|c|c|c|c|c|c|c|c|}
\hline $\mathrm{PB} \# \mathrm{VAR}$ & SMODELS & $\operatorname{MODELS}_{c c}$ & ASSAT & DLV & ulv & flv & flu & fbu \\
\hline 14300 & 1.2 & 7.23 & $\underline{0.85}$ & 2.55 & 0.59 & $\underline{0.8}$ & 1.5 & 1.37 \\
\hline 24.5300 & 39.97 & TIME & TIME & 130.49 & TIME & TIME & 115.29 & $\underline{40.38}$ \\
\hline $\begin{array}{lll}3 & 5 & 300\end{array}$ & 7.57 & 149.37 & TIME & 26.78 & 456.22 & 538.89 & 17.64 & $\underline{11.32}$ \\
\hline 45.5300 & 2.26 & 33.12 & 94.78 & 7.37 & 72.83 & 53.26 & $\underline{4.42}$ & $\underline{3.59}$ \\
\hline $\begin{array}{lll}5 & 6 & 300\end{array}$ & 1.05 & 12.72 & 22.5 & 3.26 & 24.73 & 21.89 & $\underline{1.83}$ & $\underline{1.63}$ \\
\hline $\begin{array}{lll}6 & 4 & 350\end{array}$ & $\underline{4.11}$ & 12.6 & 13.4 & 49.3 & 2.2 & 5.74 & 11.48 & 8.85 \\
\hline 74.5350 & 318.1 & TIME & TIME & TIME & TIME & TIME & TIME & $\underline{384.66}$ \\
\hline $8 \quad 5350$ & 44.2 & TIME & TIME & 147.16 & TIME & TIME & 134.34 & $\underline{54.07}$ \\
\hline 95.5350 & 12.66 & 252.11 & TIME & 32.07 & TIME & 506.08 & $\underline{20.37}$ & $\underline{13.61}$ \\
\hline $10 \quad 6 \quad 350$ & 3.37 & 37.99 & 174.61 & 8.76 & 95.61 & 104.36 & $\underline{6.05}$ & $\underline{4.86}$ \\
\hline 114200 & $\underline{3.3}$ & $\underline{2.02}$ & $\underline{2.44}$ & 32.39 & 5.34 & $\underline{3.32}$ & $\underline{1.93}$ & 1.75 \\
\hline 124.5200 & 6.84 & 1.7 & 3.28 & 83.63 & 6.15 & 5.82 & $\underline{2.09}$ & $\underline{1.93}$ \\
\hline $13 \quad 5 \quad 200$ & 22.8 & 2.5 & 8.21 & 82.97 & 9.82 & 9.02 & $\underline{3.88}$ & $\underline{3.33}$ \\
\hline 145.5200 & 9.42 & 1.76 & 4.14 & 39.47 & 7.5 & 6.38 & $\underline{2.97}$ & $\underline{2.85}$ \\
\hline $15 \quad 6 \quad 200$ & 8.12 & 0.85 & $\underline{1.4}$ & 23.93 & 3.24 & 2.95 & $\underline{1.25}$ & $\underline{1.53}$ \\
\hline $16 \quad 4 \quad 300$ & 298.67 & 73.64 & 234.09 & TIME & 265.43 & 218.48 & $\underline{41.97}$ & 31.05 \\
\hline 174.5300 & TIME & TIME & TIME & TIME & TIME & TIME & 190.73 & 135.11 \\
\hline $18 \quad 5 \quad 300$ & TIME & 412.69 & TIME & TIME & TIME & TIME & 136.67 & 99.75 \\
\hline 195.5300 & TIME & 233.72 & TIME & TIME & TIME & TIME & 129.29 & 78.63 \\
\hline $\begin{array}{lll}20 & 6 & 300\end{array}$ & TIME & 191.62 & TIME & TIME & TIME & TIME & 107.34 & 65.83 \\
\hline
\end{tabular}

2. Lines (11)-(20) correspond to programs randomly generated according to the methodology proposed in (Lin and Zhao, 2003b). Given a set $P$ with $N$ atoms and a positive number $k$, a randomly generated rule has

a) the head which is randomly selected from $P$, and

b) the body consisting of $k-1$ different atoms, each randomly selected from $P$ and negated with probability 0.5 .

A randomly generated program with $L$ rules consists of $L$ randomly generated distinct rules. In general these randomly generated programs are non tight. 
Both categories of problems have been generated with $k=3$ and $L$ varying from $0.5 \times N$ to $12 \times N$ with step 0.5 . $N$ has been fixed to 300 and 350 for the instances being the translation of $k$-SAT problems, and to 200 and 300 for the instances generated according to Lin and Zhao's methodology.

For each ratio $L / N$ (indicated in the column "PB"), we generated 10 instances, and the table presents the median results for the most difficult 5 ratios (the other being quite easily solved by all the systems).

On these benchmarks fbu has the overall best performances: it is almost always the fastest system or within a factor of 2 from the fastest. SMODELS is faster than fbu in the median case when considering the translation of $k$-SAT instances. However, on these benchmarks, SMODELS times out on 2 programs when $N=300$, while fbu times out only on 1 program. ${ }^{6}$ SMODELS' good performances on these benchmarks are not surprising given that also SMODELS implements failed literal detection, together with a heuristic similar to our unit heuristic. However, considering the programs generated according to Lin and Zhao's methodology, we see that SMODELS is not competitive with fbu which (together with flu) scales much better than all the rival systems.

Considering CMODELS2's combinations, fbu is the fastest (confirming expectations), but also flu performs quite well. Coupling these facts with the bad performances of flv, it emerges that the unit heuristic is very effective on these benchmarks and makes learning useless.

\subsection{LARGE PROGRAMS}

Table II shows the results when considering large (i.e., with approximately 10.000 or more atoms) programs. As in the previous subsection, the table is divided in two parts:

1. Programs (21)-(26) are tight: In particular (21)-(23) and (24)-(26) encode respectively blocks world planning and 4-colorability problems in a graph with $V$ vertexes. $V$ is the number in the label " $4 \mathrm{c} V$ " in column PB. All the tight programs but bw*e9 have answer sets and are available at SMODELS' web site.

2. Programs (27)-(39) are non tight. In particular, we consider Hamiltonian circuit problems on complete graphs, using both the basic encoding of Niemela (1999) (programs (27)-(31)), and the non basic encoding (programs (32)-(36)) from http://www.cs .engr.uky.

\footnotetext{
${ }^{6}$ Increasing $N$ to 400 we get the same picture: SMODELS is faster than fbu in the median case, but it times out on 11 programs, while fbu times out on 10 . We decided not to show the results for $N=400$ because most of the other solvers times out also in the median case for most of the ratios $L / N$.
} 
Table II. Performances on large programs. Problems (21)-(26) are tight. Problems (27)-(39) are non tight.

\begin{tabular}{|c|c|c|c|c|c|c|c|c|c|}
\hline PB & \#VAR & SMODELS & $\operatorname{SMODELS}_{c c}$ & ASSAT & DLV & ulv & flv & flu & fbu \\
\hline $21 \mathrm{bw}^{*} \mathrm{~d} 9$ & $9956+$ & 6.76 & 7.63 & $\underline{1.72}$ & & 1.02 & 5.84 & 2.69 & 2.75 \\
\hline $22 \mathrm{bw}^{*} \mathrm{e} 9$ & 12260 & 4.3 & 4.51 & 4.22 & & 0.98 & $\underline{1.91}$ & $\underline{1.92}$ & $\underline{1.93}$ \\
\hline $23 \mathrm{bw}^{*} \mathrm{e} 10$ & $13482+$ & 11.15 & 12.43 & 2.66 & & 1.29 & 7.51 & 5.03 & 4.95 \\
\hline 24 4c1000 & $14955+$ & 22.28 & 4.95 & $\underline{0.6}$ & & 0.48 & 37.86 & 15.41 & 15.23 \\
\hline $254 \mathrm{c} 3000$ & $44961+$ & 202.84 & 1143.13 & 2.19 & & 8.86 & 369.27 & 144.12 & 142.83 \\
\hline $264 \mathrm{c} 6000$ & $89951+$ & 856.13 & TIME & 14.85 & & 99.50 & TIME & 583.55 & 578.98 \\
\hline $27 \mathrm{np} 60 \mathrm{c}$ & $10742+$ & 242.61 & 30.81 & 84.87 & 361.80 & 2.83 & 1611.32 & 44.12 & 44.11 \\
\hline $28 \mathrm{np} 70 \mathrm{c}$ & $14632+$ & 557.08 & 55.31 & 520.80 & 798.96 & 4.69 & TIME & 97.44 & 97.87 \\
\hline $29 \mathrm{np} 80 \mathrm{c}$ & $19122+$ & 1001.88 & 90.59 & 53.25 & 1587.60 & 7.2 & TIME & 195.08 & 190.49 \\
\hline $30 \mathrm{np} 90 \mathrm{c}$ & $24212+$ & 2064.61 & 144.72 & 1416.24 & 2807.84 & 10.42 & TIME & 364.54 & 357.92 \\
\hline $31 \mathrm{np} 100 \mathrm{c}$ & $29902+$ & 3573.19 & 215.37 & TIME & TIME & 14.23 & TIME & 610.2 & 608.96 \\
\hline 32 np60c & $10683+$ & $\underline{7.05}$ & $\underline{3.82}$ & & & 3.55 & 340.86 & 8.03 & 7.82 \\
\hline $33 \mathrm{np} 70 \mathrm{c}$ & $14563+$ & 15.67 & 5.92 & & & $\underline{10.54}$ & 782.69 & 15.39 & 14.92 \\
\hline $34 \mathrm{np} 80 \mathrm{c}$ & $19043+$ & 32.29 & 9.01 & & & $\underline{15.05}$ & 1538.86 & 23.63 & 25.94 \\
\hline $35 \mathrm{np} 90 \mathrm{c}$ & $24123+$ & 53.21 & 14.13 & & & 32.19 & 2918.82 & 38.75 & 50.08 \\
\hline $36 \mathrm{np} 100 \mathrm{c}$ & $29803+$ & 83.11 & 14.95 & & & 34.18 & TIME & 59.15 & 62.64 \\
\hline 37 mutex 4 & $14698+$ & 14.14 & 5.35 & $\underline{0.54}$ & 367.89 & 0.46 & 28.29 & 28.3 & 28.26 \\
\hline 38 mutex 3 & $278074+$ & $\underline{163.94}$ & 110.27 & MEM & & TIME & TIME & TIME & TIME \\
\hline 39 phi3 & $16930+$ & 3.23 & 3.04 & 53.28 & & 1.43 & 55.62 & 12.15 & TIME \\
\hline
\end{tabular}

edu/ai/benchmark-suite/ham-cyc.sm. The remaining 3 programs in the table are related to the problem of checking requirements in a deterministic automaton and are described in (Ştefănescu et al., 2003). The first of these 3 programs is the biggest instance in the suite of the "IDFD" problems, while the other two programs belong to the "Morin" suite.

Overall, the picture that emerges is that ulv is the fastest system: Even though SMODELS is the only system that never times out, it is far slower than ulv (and other systems as well) on many problems. The good performances of ulv are particularly remarkable given that the test suite contains Hamiltonian circuit problems, and these benchmarks have exponentially many loops. Thus, one would expect these problems to be difficult for ASSAT, but also for all CMODELS2 versions in the case 
it will generate and then reject (exponentially) many candidate answer sets. As it can be observed, this is not the case, at least for ulv. Finally, the table also shows an instance on which ASSAT blows up in memory: As a matter of facts, ASSAT exceeds all the available memory also on other instances, here not shown because all the other systems time out on them.

Considering the different CMODELS2 versions - beside the fact that ulv is the best version - by comparing ulv and flv we see that adding failed-literal usually causes a significant degradation in the performances. These results match the expectations. Indeed, ulv (and also ASSAT) uses a MCHAFF-like solver and performs a few operations at each (branching) node: For (very) large programs, even a linear-time (in the number of atoms) operation can be prohibitive if performed at each branching node. Interestingly, considering flv, flu and fbu we see that it is almost always the case that the last system performs better than the second, and that the second is better than the first. On these benchmarks, adding learning to a look-ahead solver does not help. However, the gap between fbu and flu is not big. Thus, adding learning to fbu does not help, but does not hurt too much: we believe that this is due to the lazy data structures used by all the CMODELS2 versions, which are fundamental to keep low the burden of managing learned clauses.

\subsection{NON RANDOM, NON LARGE PROGRAMS}

Table III contains the results on non random, non large logic programs. In more details, ${ }^{7}$

1. Benchmarks (40)-(48) and (73)-(77) are respectively tight and non tight bounded model checking (BMC) problems of asynchronous concurrent systems, as described in (Heljanko and Niemelä, 2003). These problems are about proving properties in a given number of steps, represented as the last number in the instance name.

2. Benchmarks (49)-(54) are about the Schur numbers problem, expressed as basic (49)-(51) and non basic (52)-(54) programs respectively. The label "schurX.K-N" refers to a problem where, given a positive integer $n$, the set of integers $\mathrm{N}$ defined as $\mathrm{N}=\{1,2, \ldots n\}$ has to be partitioned into $\mathrm{K}$ bins such that each bin is sum-free,

\footnotetext{
7 Benchmarks (40)-(48), (73)-(77) or the generator are available at http: //www.tcs.hut.fi/ ^kepa/experiments/boundsmodels/. Benchmarks (49)-(57) are available at the ASPARAGUS web page http://asparagus.cs.uni-potsdam.de/. Benchmarks (58)-(60) belong to the SMODELS test suite and are publicly available at http://www.tcs.hut.fi/Software/smodels/tests/, encoding by Niemela (1999).
} 
Table III. Performances on non random, non large programs. Benchmarks (40)-(60) are tight, while the others are non tight.

\begin{tabular}{|c|c|c|c|c|c|c|c|c|c|}
\hline $\mathrm{PB}$ & \#VAR & SMODELS & SMODELS $_{c c}$ & ASSAT & DLV & ulv & flv & flu & fbu \\
\hline $40 \quad \mathrm{~d}^{*} 12 * \mathrm{i} * 9$ & 1186 & $\underline{368}$ & $\underline{435.48}$ & & & 223.93 & $\underline{290.15}$ & $\underline{353.53}$ & TIME \\
\hline $41 \quad \mathrm{k}^{*} \mathrm{i}^{*} 29$ & 3199 & 990.95 & 20.88 & & & 415.54 & 204.87 & 44.14 & 589.45 \\
\hline $42 \quad \mathrm{k}^{*} \mathrm{~s} * 29$ & 3169 & 909.46 & 16.89 & & & 353.69 & 1028.77 & 59.99 & TIME \\
\hline $43 \mathrm{~m}^{*} 3 * \mathrm{i} * 10$ & $1933+$ & 10.98 & 1.65 & & & 16.23 & 32.23 & 26.71 & 16.55 \\
\hline $44 \mathrm{~m}^{*} 4^{*} \mathrm{i}^{*} 12$ & $3475+$ & 1132.16 & 3.82 & & & 1063.15 & 867.49 & TIME & 3229.09 \\
\hline $45 \mathrm{~m} * 4 * \mathrm{~s} * 8$ & $1586+$ & 89.26 & 1.3 & & & 17.02 & 27.59 & 421.30 & 327.55 \\
\hline $46 \quad \mathrm{q}^{*} \mathrm{i}^{*} 17$ & 2201 & 517.64 & 53.71 & & & 1539.96 & 505.15 & 259.05 & 816.26 \\
\hline $47 \quad \mathrm{e}^{*} 3 * \mathrm{i} * 15$ & $7832+$ & 35.58 & 77.02 & & & 479.28 & TIME & $\underline{7.15}$ & 6.87 \\
\hline $48 \quad \mathrm{e}^{*} 4 *^{*} *^{*} 13$ & 6447 & 221.18 & 56.21 & & & 87.63 & 567.27 & 20.02 & 19.41 \\
\hline 49 schur1.4-43 & $736+$ & 0.43 & 0.95 & $\underline{0.67}$ & 590.57 & 1.4 & 2.07 & 0.82 & 0.88 \\
\hline 50 schur1.4-44 & $753+$ & 0.44 & 91.25 & 1.07 & TIME & 5.97 & 5.62 & 92.63 & 43.01 \\
\hline 51 schur1.4-45 & 770 & 571.17 & 1110.68 & 434.93 & TIME & $\underline{229.04}$ & 417.34 & 244.35 & 116.51 \\
\hline 52 schur $2.4-43$ & $564+$ & 0.33 & $\underline{0.56}$ & & & 1.27 & 1.04 & $\underline{0.4}$ & $\underline{0.38}$ \\
\hline 53 schur2.4-44 & $577+$ & 82.72 & 47.78 & & & 6.14 & 2.8 & 47.99 & 18.93 \\
\hline 54 schur $2.4-45$ & 590 & 578.73 & 672.86 & & & 226.69 & 392.78 & 148.39 & 63.2 \\
\hline $55 \quad 15$ puz. 18 & $5945+$ & 17.55 & 6.94 & $\underline{1.06}$ & 141.68 & 0.98 & 2.9 & 9.85 & 9.24 \\
\hline $56 \quad 15$ puz.19 & $6258+$ & 20.94 & 7.14 & 3.61 & 208.41 & 1.35 & 2.93 & 11.65 & 10.76 \\
\hline 57 15puz.20 & 6571 & 70.27 & 8.22 & 4.59 & TIME & 1.28 & 10.22 & 64.54 & 82.68 \\
\hline 58 pige.9.10 & 210 & 44.77 & 65.91 & 1.1 & & $\underline{1.26}$ & 4.33 & 1259.84 & $=32.06$ \\
\hline 59 pige. 10.11 & 253 & 484.63 & 1029.38 & $\underline{23.83}$ & & 12.41 & 55.46 & TIME & 339.06 \\
\hline 60 pige. 51.50 & $5252+$ & 106.79 & 24.29 & $\underline{2.49}$ & & 1.63 & 221.33 & 6.85 & 7.26 \\
\hline $8 \mathrm{i}-1$ & 2329 & 7.48 & 7.17 & $\underline{0.86}$ & & 0.49 & $\underline{0.85}$ & $\underline{0.84}$ & $\underline{0.81}$ \\
\hline $11 \mathrm{i}-1$ & 4760 & 36.18 & 35.53 & 3.15 & & 1.64 & 4.92 & $\underline{2.47}$ & $\underline{2.44}$ \\
\hline $8 \mathrm{i}$ & $2627+$ & 17.35 & 9.30 & $\underline{0.98}$ & & 0.63 & 1.27 & $\underline{0.89}$ & $\underline{0.88}$ \\
\hline $11 \mathrm{i}$ & $5301+$ & 37.71 & 43.90 & $\underline{3.59}$ & & 2.16 & 15.55 & 6.07 & 5.79 \\
\hline $8 \mathrm{i}+1$ & $2925+$ & 12.08 & 15.17 & 1.09 & & $\underline{1.34}$ & 4.31 & $\underline{1.34}$ & $\underline{1.37}$ \\
\hline $11 \mathrm{i}+1$ & $5842+$ & 54.30 & 62.39 & $\underline{3.9}$ & & 2.49 & 24.27 & 22.01 & 19.71 \\
\hline $8 \mathrm{i}-1$ & 1897 & 0.53 & 0.66 & & & 0.15 & $\underline{0.29}$ & $\underline{0.27}$ & $\underline{0.27}$ \\
\hline $11 \mathrm{i}-1$ & 3812 & 1.6 & 1.96 & & & 0.39 & 1.71 & $\underline{0.75}$ & $\underline{0.7}$ \\
\hline $8 \mathrm{i}$ & $2132+$ & 0.76 & 0.8 & & & 0.22 & $\underline{0.42}$ & $\underline{0.27}$ & $\underline{0.3}$ \\
\hline $11 \mathrm{i}$ & $4233+$ & 1.85 & 2.57 & & & 0.52 & 6.76 & 1.9 & 1.88 \\
\hline $8 \mathrm{i}+1$ & $2367+$ & 1.8 & 1.05 & & & $\underline{0.68}$ & 1.65 & 0.47 & $\underline{0.49}$ \\
\hline $72 \quad 11 \mathrm{i}+1$ & $4654+$ & 2.5 & 4.12 & & & 0.6 & 10.42 & 5.26 & 5.21 \\
\hline $73 \mathrm{~d}^{*} 10^{*} \mathrm{i}^{*} 12$ & $1488+$ & 132.72 & 2.25 & & & 488.76 & 1212.89 & 152.8 & TIME \\
\hline 74 d*10*s*9 & $1140+$ & 9.75 & 3.11 & & & 6.38 & 19.31 & 87.64 & TIME \\
\hline $75 \mathrm{~d}^{*} 12 * \mathrm{~s}^{*} 10$ & $1511+$ & 296.45 & 1.1 & & & 53.2 & 165.9 & 733.9 & TIME \\
\hline $76 \quad \mathrm{~d}^{*} 8 * \mathrm{i} * 10$ & $1003+$ & $\underline{1.76}$ & 2.42 & & & 12.28 & 25.03 & 1.21 & 11.86 \\
\hline $77 \quad d^{*} 8 * s^{*} 8$ & $819+$ & 0.73 & 0.14 & & & 0.47 & 3.73 & 2.38 & 1221.53 \\
\hline
\end{tabular}


i.e., for each $Z \in N$ and $Y \in N(i) Z$ and $Z+Z$ are in different bins, and $(i i)$ if $\mathrm{Z}$ and $\mathrm{Y}$ are in the same bin, then $\mathrm{Z}+\mathrm{Y}$ is in a different bin. We denote with $\mathrm{X}=1$ the basic encoding and with $\mathrm{X}=2$ the non basic encoding

3. Benchmarks (55)-(57) are programs encoding the 15 puzzle problem. In a label "15puz.M", M denoted the number of moves in which the final configuration has to be reached. The initial configuration is not fixed and varies from program to program.

4. Benchmarks (58)-(60) are tight programs encoding pigeons problems. In a label "pige.h.p", $h$ denotes the number of holes and $p$ the number of pigeons.

5. Benchmarks (61)-(72) are blocks world planning problems encoded as basic programs in lines (61)-(66), and as non basic programs in lines (67)-(72)), the formulations due to Erdem (2002). In the tables, in the column PB the "8" or " 11 " represents the number of blocks; while an " $i$ " (standing for "number of steps") means that the instance corresponds to the problem of finding a plan in " $i$ " steps, where " $i$ " is the minimum integer for which a plan exists. Thus, the instances with " $i$ " and " $i+1$ " in the label admit at least one answer set, while those with " $i-1$ " do not have answer sets. Technically speaking, these programs are non tight. However, these problems are "tight on their completion models" (Babovich et al., 2000): If $\Pi$ is one such program, each model of the completion of $\Pi$ is guaranteed to be also an answer set of $\Pi$.

For these benchmarks results are mixed: On BMC problems, SMODELS ${ }_{c c}$ has the best performances overall, while on the other benchmarks it is ulv which has the best performances overall. What is most interesting is that there is no version of CMODELS2 dominating the others on the

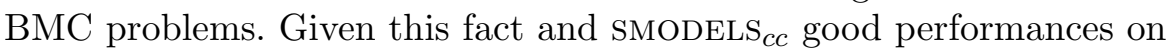
$\mathrm{BMC}$ instances, we believe that on non random, non large problems the "overall best" solver is somewhere in between ulv and fbu, i.e., that it can can be obtained by adding a little bit of failed-literal detection to ulv. This can be done is several ways, e.g., by checking if a literal is failed only if it belongs to a pool of "most promising" literals (as, e.g., it is done by SATZ), or by checking all the literals but not at each branching node. All of this is subject of future research.

It is also worth noting that, overall, flu is better than flv: This can be explained by the bad interaction between failed-literal and VSIDS. For non random, large formulas, this phenomena was already showed to hold in SAT (Giunchiglia et al., 2003). 


\subsection{CMOdels2 And The Other Systems}

Given the results of the experimental analysis, we now sum up what we consider to be the advantages and disadvantages of each system we considered, both from a theoretical and a practical point of view, when compared to CMODELS2.

SMODELS (Simons et al., 2002). SMODELS is a system for non disjunctive answer set programming. Its algorithm has been inspired by Davis-Logemann-Loveland procedure, and incorporates powerful pruning techniques.

SMODELS is also the basic engine for the solver for disjunctive logic programming called GNT (Janhunen and Niemelä, 2004; Janhunen et al., 2005). A key feature of SMODELs is that it is a native system, i.e., it works directly on the input logic program. Because of this, it can take advantage of the structure of the program, e.g., by keeping more compact representations of the rules than CMODELS2, which compiles down everything to a set of clauses. However, it does not incorporate some of the most recent advances, e.g., learning. The experimental results for SMODELS are still positive overall, being among the best solvers in all the categories of problems we considered.

SMODELS $_{c c}$ (Ward and Schlipf, 2004). SMODELS $c$ is SMODELS enhanced with clause-learning (Moskewicz et al., 2001) and a BERKMIN-like heuristic (Goldberg and Novikov, 2003). SMODELS sc $_{c}$ inherits from SMODELS its compact data-structures for rules. However, due to such compactness, the incorporation of learning in SMODELS required the construction of an implication graph, and this operation turned out to be relatively complex and costly when compared to the analogous construction in a SAT solver. Indeed, SMODELS ${ }_{c c}$ cannot deal with programs containing weight constraint rules, and this also witnesses the difficulty of implementing learning on top of SMODELS compact data structures for such rules. On the other hand, learning comes for free with our approach. Further, with relatively little additional programming effort, our procedure can be based on the latest SAT tools. We used our tool SIMO to validate the viability and effectiveness of the approach, and obtained a solver with, e.g., learning and unit propagation based on lazy data structures using a two literal watching schema. Modifying SMODELS or SMODELS $c c$ in order to use lazy data structures would require a rewriting of significant portions of the solvers. From a practical point of view, SMODELS $c$ is quite effective, especially on some classes of non tight programs. 
DLV (Leone et al., 2005). DLV is the state-of-the-art system in disjunctive logic programming, with techniques especially tailored for this class of programs. Also DLV is a native system and its algorithm is based on the Davis-Logemann-Loveland procedure.

However, since it can deal with the more expressive class of disjunctive programs, it needs a co-NP check to test if a candidate model is indeed an answer set. The check is performed only if needed: In the case of non disjunctve programs (the ones this paper faces), it is not applied.

DLV has same peculiarities: During the computation, it uses a fourvalued interpretations for atoms. The truth values considered are True, False, "undefined" and "must be true"; a "must be true" atom is like an atom assigned to True but it is missing a "supporting" rule that must be determined later on. Moreover, DLV heuristic is guided by a preselected list of literals (PT-literals) with the aims of maintaining the candidate model as minimal as possible. DLV key strength is that it can deal with disjunctive logic programs. However, on the restricted class of non disjunctive logic programs, its performances are not impressive, at least on the benchmarks that we considered.

ASSAT (Lin and Zhao, 2002; Lin and Zhao, 2004). ASSAT has been the first ASP SAT-based system non restricted to tight programs. The SAT solver is used as a black box and thus ASSAT inherits all the optimizations implemented in it. ASSAT uses MCHAFF (Moskewicz et al., 2001) as SAT solver. As we have seen, ASSAT is quite effective especially on non random programs. From a theoretical perspective, the main drawback of ASSAT is that it is not guaranteed to work in polynomial space. This fact also emerges in some of the benchmarks that we considered and for which ASSAT exhausted all the available memory. From a practical point of view, ASSAT is limited to basic programs and cannot handle choice, cardinality and weight constraint rules.

CModels2. Cmodels2 is a SAT-based system designed after ASSAT in order to solve its theoretical drawbacks. CMODELS2 incorporates various solvers. fbu is our default choice for randomly generated programs, and ulv is our default for non random programs. The experimental analysis showed that on random problems fbu has the best performances overall of all the solvers that we considered, and the same holds for ulv when considering large problems. On the other benchmarks, ulv is competitive with the best of the other solvers. These results show the effectiveness of our SAT-based approach. These results are particularly remarkable given that our two solvers implement relatively simple SAT 
strategies, if compared to the ones that are now available, some of which already incorporated by various answer set solvers. For instance, ulv uses MCHAFF heuristic, while Berkmin heuristic (used by SMODELS Sc $_{c}$ ) is considered to be better. In fbu each not yet assigned literal is checked to see if it is failed, and these checks are performed before each branching: SMODELS and SMODELS $c c$ implement the correspondent strategy of failed-literal, but they check only a subset of the unassigned literals (and the unchecked are guaranteed to be not failed). We expect that the incorporation of Berkmin heuristic and SMODELS failed literal detection strategy in ulv and fbu respectively will lead to further improvements in the performances when run on the respective application domains.

\section{Conclusions and future work}

We have presented a SAT-based procedure that $(i)$ can deal with any logic program (ii) works on a propositional formula without additional variables except for those introduced during the clause form conversion, (iii) is guaranteed to work in polynomial space. Furthermore, ASPSAT can be easily modified in order to compute all answer sets (still working in polynomial space). We have shown how to implement ASPSAT on top of current state-of-the-art solvers with/without learning. The experimental evaluation shows that:

1. CMOdELs2 is competitive with other state-of-the-art systems;

2. depending on the type of program different search strategies are best.

This suggests that future development of answer set solvers should be done by focusing on certain classes of problems. In our analysis we identified two classes of programs that need completely different strategies, i.e., random and large programs. This also implies that benchmarking should be done by considering the application domain which they have been developed for. This reflects what is nowadays a standard in the SAT competition, where there is a track for solvers designed for random problems, and a separate track for solvers designed for large industrial benchmarks. Solvers get designed and specialized for one track, and indeed the top performers in one track behave very badly in the other.

Considering the future, there are several directions in which this work can be improved.

First CMODELS2 can be improved as a solver for non disjunctive programs. This can be done by improving the SAT solving part, i.e., DLL, or the checking procedure, i.e., test. 
As anticipated in the previous section, we believe that DLL performances can be improved by implementing better failed literal detection strategies and/or heuristics. About the heuristics - besides those derived from the SAT literature as BERKMIN's - we believe that it is possible to design heuristics tailored for answer set solving. One such heuristic assigns atoms to False while branching: Intuitively, we would like to generate assignments with as many atoms as possible assigned to False, thus going through minimality. A first, simple implementation of this heuristic, produces dramatic speed-ups on some domains (for instance, ulv is able to solve all the non tight problems in Table II in a few seconds, including the mutex 3 instance, i.e., the only instance on which ulv times out), but it seems to badly interact with learning in some other domains. Another possibility is to incorporate another SAT solver with the latest advancements, e.g., MiniSAT (Eén and Sörensson, 2003) the winner of the last SAT competition.

Considering the checking procedure test, recently Gebser and Schaub (2005) introduced the notion of "active elementary loop with respect to an assignment $S "$, and they showed that the corresponding loop formula is falsified by $S$, like the formula associated to a maximal terminating loop. One crucial difference between an active elementary loop and a maximal terminating one is that no sub-loop of an active elementary loop is also falsified by $S$. A maximal terminating loop on the other hand is not always an active elementary loop of the program. It is still an open question whether the use of active elementary loops in SAT-based procedures like CMODELS2 or ASSAT improves their performances.

Another direction of work is to extend CMODELS2 ideas in order to deal with disjunctive logic programming where, as for DLV, the co-NP check involves the use of a SAT solver. A preliminary implementation and analysis are encouraging (Lierler, 2005), but more work has to be done in order to improve the overall efficiency of the solver.

\section{Acknowledgements}

We are grateful to Paolo Ferraris, Nicola Leone, Vladimir Lifschitz and the anonymous reviewers of this paper for their helpful comments and/or discussions on the subject of the paper; to Esra Erdem and Keijo Heljanko for providing us with the benchmarks; and to Francesco Calimeri for his support on DLV. This work is partially supported by MIUR (Italian Ministry of Education, University and Research) and Texas Higher Education Coordinating Board under Grant 003658-03222001. 


\section{References}

Armando, A., C. Castellini, and E. Giunchiglia: 1999, 'SAT-based procedures for temporal reasoning'. In: Lecture Notes in Computer Science, Vol. 1809. pp. 97-108.

Armando, A., C. Castellini, E. Giunchiglia, and M. Maratea: 2005, 'The SAT-based Approach to Separation Logic'. Journal of Automated Reasoning. To appear.

Babovich, Y., E. Erdem, and V. Lifschitz: 2000, 'Fages' Theorem and Answer Set Programming'. In: Proc. NMR.

Baral, C. Gelfond, M. and R. Scherl: 2004, 'Using answer set programming to answer complex queries'. In: Workshop on Pragmatics of Question Answering at HLTNAAC2004.

Barrett, C. W., D. L. Dill, and A. Stump: 2002, 'Checking Satisfiability of FirstOrder Formulas by Incremental Translation to SAT'. In: E. Brinksma and K. G. Larsen (eds.): 14th International Conference on Computer Aided Verification (CAV), Vol. 2404 of Lecture Notes in Computer Science. pp. 236-249, Springer. Copenhagen, Denmark.

Bayardo, Jr., R. J. and R. C. Schrag: 1997, 'Using CSP Look-Back Techniques to Solve Real-World SAT Instances'. In: Proceedings of the 14th National Conference on Artificial Intelligence and 9th Innovative Applications of Artificial Intelligence Conference (AAAI-97/IAAI-97). Menlo Park, pp. 203-208, AAAI Press.

Ben-Eliyahu, R. and R. Dechter: 1996, 'Propositional Semantics for Disjunctive Logic Programs'. Annals of Mathematics and Artificial Intelligence 12, 53-87.

Clark, K.: 1978, 'Negation as failure'. In: H. Gallaire and J. Minker (eds.): Logic and Data Bases. New York: Plenum Press, pp. 293-322.

Ştefănescu, A., J. Esparza, and A. Muscholl: 2003, 'Synthesis of Distributed Algorithms Using Asynchronous Automata'. In: Proceedings of CONCUR'03, Vol. 2761. pp. 27-41, Springer.

Davis, M., G. Logemann, and D. W. Loveland: 1962, 'A machine program for theorem proving'. Communication of ACM 5(7), 394-397.

de Moura, L., H. Rueß, and S. Sorea: 2002, 'Lazy Theorem Proving for Bounded Model Checking over Infinite Domains'. In: A. Voronkov (ed.): Automated Deduction - CADE-18, Vol. 2392 of Lecture Notes in Computer Science. pp. 438-455, Springer-Verlag.

Dixon, H. E., M. L. Ginsberg, E. M. Luks, and A. J. Parkes: 2004, 'Generalizing Boolean Satisfiability II: Theory.'. J. Artif. Intell. Res. (JAIR) 22, 481-534.

Dowling, W. and J. Gallier: 1984, 'Linear-time algorithms for testing the satisfiability of propositional Horn formulae'. Journal of Logic Programming 3, 267-284.

Eén, N. and N. Sörensson: 2003, 'An Extensible SAT-solver'. In: Theory and Applications of Satisfiability Testing, 6th International Conference, SAT 2003. Santa Margherita Ligure, Italy, May 5-8, 2003 Selected Revised Papers. pp. 502-518.

Erdem, E.: 2002, 'Theory and applications of answer set programming'. Ph.D. thesis, University of Texas at Austin.

Erdem, E. and V. Lifschitz: 2001, 'Fages' theorem for Programs with Nested Expressions'. In: Proc. International Conference on Logic Programming. pp. $242-254$.

Faber, W., N. Leone, and G. Pfeifer: 2001, 'Experimenting with Heuristics for Answer Set Programming.'. In: IJCAI. pp. 635-640.

Fages, F.: 1994, 'Consistency of Clark's completion and existence of stable models'. Journal of Methods of Logic in Computer Science 1, 51-60. 
Ferraris, P. and V. Lifschitz: 2005, 'Weight constraints as nested expressions'. Theory and Practice of Logic Programming 5, 45-74.

Gebser, M. and T. Schaub: 2005, 'Loops: Relevant or Redundant?'. In: Proc of 8th International Conference on Logic Programming and Nonmonotonic Reasoning. pp. 53-65, Springer-Verlag.

Gelfond, M. and V. Lifschitz: 1988, 'The stable model semantics for logic programming'. In: R. Kowalski and K. Bowen (eds.): Logic Programming: Proc. Fifth Int'l Conf. and Symp. pp. 1070-1080.

Gelfond, M. and V. Lifschitz: 1991, 'Classical negation in logic programs and disjunctive databases'. New Generation Computing 9, 365-385.

Gent, I., H. V. Maaren, and T. Walsh (eds.): 2000, SAT2000. Highlights of Satisfiability Research in the Year 2000. IOS Press.

Giunchiglia, E., F. Giunchiglia, and A. Tacchella: 2002, 'SAT-Based Decision Procedures for Classical Modal Logics'. Journal of Automated Reasoning 28, 143-171. Reprinted in (Gent et al., 2000).

Giunchiglia, E. and M. Maratea: 2005a, 'Evaluating Search Strategies and Heuristics for Efficient Answer Set Programming'. In: Advanced in Artificial Intelligence: Conference of the Italian Association for Artificial Intelligence, $A I^{*} I A$ '05, Milan, Italy, September 20-23, 2005: proceedings. pp. 37-51, Springer.

Giunchiglia, E. and M. Maratea: 2005b, 'On the relation between SAT and ASP procedures (or, between smodels and cmodels)'. In: Proc. of the 21th International Conference on Logic Programming (ICLP). pp. 37-51, Springer.

Giunchiglia, E., M. Maratea, and Y. Lierler: 2004, 'SAT-Based Answer Set Programming'. In: Proceedings of the Nineteenth National Conference on Artificial Intelligence, Sixteenth Conference on Innovative Applications of Artificial Intelligence, July 25-29, 2004, San Jose, California, USA. AAAI Press / The MIT Press.

Giunchiglia, E., M. Maratea, and A. Tacchella: 2003, '(In)Effectiveness of LookAhead Techniques in a Modern SAT Solver'. In: 9th International Conference on Principles and Practice of Constraint Programming (CP-03). pp. 842-846.

Giunchiglia, E., M. Maratea, A. Tacchella, and D. Zambonin: 2001, 'Evaluating Search Heuristics and Optimization Techniques in Propositional Satisfiability.' In: Automated Reasoning, First International Joint Conference (IJCAR), Vol. 2083 of Lecture Notes in Computer Science. pp. 347-363, Springer Verlag.

Goldberg, E. and Y. Novikov: 2003, 'BerkMin: A fast and robust SAT solver'. In: Proc. of the Design, Automation and Test in Europe Conference and Exposition 2003. pp. 142-149, IEEE Computer Society.

Heljanko, K. and I. Niemelä: 2003, 'Bounded LTL Model Checking with Stable Models'. Theory and Practice of Logic Programming 3(4\&5), 519-550. Also available as (CoRR: arXiv:cs.LO/0305040).

Janhunen, T.: 2003, 'Translatability and intranslatability results for certain classes of logic programs'. Series A: Research report 82, Helsinki University of Technology, Laboratory for Theoretical Computer Science, Espoo, Finland.

Janhunen, T.: 2004, 'Representing Normal Programs with Clauses'. In: In Proc. of 16th European Conference on Artificial Intelligence, ECAI 2004. pp. 358-362, IOS Press.

Janhunen, T. and I. Niemelä: 2004, 'GnT - A solver for disjunctive logic programming'. In: Proc. of the 7th Internation Conference on Logic Programming and Nonmonotonic Reasoning (LPNMR). pp. 331-335, Springer-Verlag. 
Janhunen, T., I. Niemelä, D. Seipel, P. Simons, and J.-H. You: 2005, 'Unfolding Partiality and Disjuntion in Stable Model Semantics'. Accepted to the ACM Transaction on Computational Logic.

Lahiri, S. K., S. A. Seshia, and R. E. Bryant: 2002, 'Modeling and Verification of Out-of-Order Microprocessors in UCLID'. In: Formal Methods in ComputerAided Design, 4th International Conference, FMCAD 2002, Portland, OR, USA, November 6-8, 2002, Proceedings. pp. 142-159.

Le Berre, D. and L. Simon: 2003, 'The essentials of the SAT'03 Competition'. In: Theory and Applications of Satisfiability Testing, 6th International Conference, SAT 2003. Santa Margherita Ligure, Italy, May 5-8, 2003 Selected Revised Papers, Vol. 2919 of LNCS.

Lee, J. and V. Lifschitz: 2003, 'Loop formulas for disjunctive logic programs'. In: Proc. ICLP-03.

Leone, N., G. Pfeifer, W. Faber, T. Eiter, G. Gottlob, S. Perri, and F. Scarcello: 2005, 'The DLV System for Knowledge Representation and Reasoning'. Accepted to ACM Transaction on Computational Logic (ToCL).

Li, C. M. and Anbulagan: 1997, 'Heuristics Based on Unit Propagation for Satisfiability Problems'. In: Proceedings of the 15th International Joint Conference on Artificial Intelligence (IJCAI-97). San Francisco, pp. 366-371, Morgan Kaufmann Publishers.

Lierler, Y.: 2005, 'Disjunctive Answer Set Programming via Satisfiability.'. In: Answer Set Programming, Vol. 142 of CEUR Workshop Proceedings.

Lierler, Y. and V. Lifschitz: 2003, 'Computing Answer Sets Using Program Completion'. Available at http://www.cs.utexas.edu/users/tag/cmodels.html.

Lifschitz, V.: 1996, 'Foundations of logic programming'. In: G. Brewka (ed.): Principles of Knowledge Representation. CSLI Publications, pp. 69-128.

Lifschitz, V. and A. Razborov: 2004, 'Why are there so many loop formulas?'. ACM Transactions on Computational Logic. To appear.

Lifschitz, V., L. R. Tang, and H. Turner: 1999, 'Nested expressions in logic programs'. Annals of Mathematics and Artificial Intelligence 25, 369-389.

Lin, F. and J. Zhao: 2003a, 'On Tight Logic Programs and Yet Another Translation from Normal Logic Programs to Propositional Logic'. In: Proc. IJCAI.

Lin, F. and Y. Zhao: 2002, 'ASSAT: Computing Answer Sets of a Logic Program by SAT Solvers'. In: Proceedings of the Eighteenth National Conference on Artificial Intelligence and Fourteenth Conference on Innovative Applications of Artificial Intelligence (AAAI/IAAI-02). Menlo Parc, CA, USA, pp. 112-118, AAAI Press.

Lin, F. and Y. Zhao: 2003b, 'Answer Set Programming Phase Transition: A study on Randomly Generated Programs'. In: Proc. ICLP.

Lin, F. and Y. Zhao: 2004, 'ASSAT: computing answer sets of a logic program by SAT solvers.'. Artificial Intelligence 157(1-2), 115-137.

Lloyd, J. and R. Topor: 1984, 'Making Prolog more expressive'. Journal of Logic Programming 3, 225-240.

Marek, V. and V. Subrahmanian: 1989, 'The Relationship Between Logic Program Semantics and Non-Monotonic Reasoning'. In: G. Levi and M. Martelli (eds.): Logic Programming: Proc. Sixth Int'l Conf. pp. 600-617.

Marek, V. and M. Truszczynski: 1999, 'Stable models as an alternative programming paradigm'. In: The Logic Programming Paradigm: a 25. Years perspective, Lecture Notes in Computer Science. Springer Verlag.

Moskewicz, M. W., C. F. Madigan, Y. Zhao, L. Zhang, and S. Malik: 2001, 'Chaff: Engineering an Efficient SAT Solver'. In: Proceedings of the 38th Design Automation Conference (DAC'01). pp. 530-535. 
Niemelä, I.: 1999, 'Logic programs with stable model semantics as a constraint programming paradigm'. Annals of Mathematics and Artificial Intelligence 25, $241-273$

Nieuwenhuis, R. and A. Oliveras: 2005, 'DPLL(T) with Exhaustive Theory Propagation and Its Application to Difference Logic.'. In: Computer Aided Verification, 17th International Conference, CAV 2005, Edinburgh, Scotland, UK, July 6-10, 2005, Proceedings. pp. 321-334.

Nogueira, M., M. Balduccini, M. Gelfond, R. Watson, and M. Barry: 2001, 'An AProlog decision support system for the space shuttle'. In: Working Notes of the AAAI Spring Symposium on Answer Set Programming.

Plaisted, D. and S. Greenbaum: 1986, 'A Structure-preserving Clause Form Translation'. Journal of Symbolic Computation 2, 293-304.

Sheridan, D.: 2004, 'The Optimality of a Fast CNF Conversion and its Use with SAT'. In: Proceedings of SAT, International Conference on Theory and Applications of Satisfiability Testing, Vancouver (Canada).

Siekmann, J. and G. Wrightson (eds.): 1983, Automation of Reasoning: Classical Papers in Computational Logic 1967-1970, Vol. 1-2. Springer-Verlag.

Silva, J. P. M. and K. A. Sakallah: 1996, 'GRASP - A new Search Algorithm for Satisfiability'. Technical report, University of Michigan.

Simons, P., I. Niemelä, and S. Timo: 2002, 'Extending and Implementing the Stable Model Semantics'. Artificial Intelligence 138(1-2), 181-234.

Syrjanen, T.: 2003, 'Lparse Manual' ${ }^{8}$.

Tseitin, G.: 1970, 'On the Complexity of Proofs in Propositional Logics'. Seminars in Mathematics 8. Reprinted in (Siekmann and Wrightson, 1983).

Ward, J. and J. S. Schlipf: 2004, 'Answer Set Programming with Clause Learning.' In: Logic Programming and Nonmonotonic Reasoning, 7th International Conference, LPNMR 2004, Fort Lauderdale, FL, USA, January 6-8, 2004, Proceedings. pp. 302-313.

Zhang, L., C. F. Madigan, M. W. Moskewicz, and S. Malik: 2001, 'Efficient Conflict Driven Learning in a Boolean Satisfiability Solver'. In: International Conference on Computer-Aided Design (ICCAD'01). pp. 279-285.

${ }^{8}$ http://www.tcs.hut.fi/Software/smodels/lparse.ps.gz . 
final.tex; 18/05/2006; $9: 11 ;$ p.42 